\title{
Single-Cell Proteomics Defines the Cellular Heterogeneity of Localized Prostate Cancer
}

Laura De Vargas Roditi ${ }^{1}$, Andrea Jacobs ${ }^{2}$, Jan H. Rueschoff ${ }^{3}$, Pete Bankhead ${ }^{4}$, Stephane Chevrier $^{2}$, Hartland W. Jackson ${ }^{2}$, , Thomas Hermanns ${ }^{5}$, Christian D. Fankhauser ${ }^{5}$, Cedric Poyet ${ }^{5}$, Felix Chun ${ }^{6}$, Niels J. Rupp ${ }^{3}$, Alexandra Tschaebunin ${ }^{7}$, Bernd Bodenmiller ${ }^{2, *}$, and Peter J. Wild $^{7,8,9, *}$

${ }^{1}$ Department of Biosystems Science and Engineering, ETH Zürich, Basel, Switzerland

${ }^{2}$ Department of Quantitative Biomedicine, University of Zürich, Zürich, Switzerland

${ }^{3}$ Department of Pathology and Molecular Pathology, University Hospital Zürich, University of Zürich, Zürich, Switzerland

${ }^{4}$ Edinburgh Pathology and Institute of Genetics and Molecular Medicine, University of Edinburgh, Edinburgh, United Kingdom

${ }^{5}$ Department of Urology, University Hospital Zurich, University of Zürich, Zürich, Switzerland

${ }^{6}$ Department of Urology, University Hospital Frankfurt, Frankfurt, Germany

${ }^{7}$ Dr. Senckenberg Institute of Pathology, University Hospital Frankfurt, Frankfurt, Germany

${ }^{8}$ Frankfurt Institute for Advanced Studies (FIAS), Frankfurt, Germany

${ }^{9}$ Wildlab, University Hospital Frankfurt MVZ GmbH, Frankfurt, Germany

*Corresponding authors:

Bernd Bodenmiller (bernd.bodenmiller@imls.uzh.ch)

Peter J. Wild (peter.wild@kgu.de) 


\section{ABSTRACT}

Localized prostate cancer exhibits multiple genomic alterations and heterogeneity at the proteomic level. Single-cell technologies capture important cell-to-cell variability responsible for

5 heterogeneity in biomarker expression that may be overlooked when molecular alterations are based on bulk tissue samples. The aim of this study was to identify novel prognostic biomarkers and describe the heterogeneity of prostate cancer and the associated immune cell infiltrates by

8 simultaneously quantifying 36 proteins using single-cell mass cytometry analysis of over 1,6 million cells from 58 men with localized prostate cancer. To perform this task, we proposed a novel computational pipeline, Franken, which showed unprecedented combination of

11 performance, sensitivity and scalability for high dimensional clustering compared to state of the 12 art methods. We were able to describe subpopulations of immune, stromal, and prostate cells, 13 including unique changes occurring in tumor tissues and high grade disease providing insights

14 into the coordinated progression of prostate cancer. Our results further indicated that men with

15 localized disease already harbor rare subpopulations that typically occur in castration-resistant 16 and metastatic disease, which were confirmed through imaging. Our methodology could be used 17 to discover novel prognostic biomarkers to personalize treatment and improve outcomes.

\section{INTRODUCTION}

The treatment of localized prostate cancer is based on clinicopathological information

22 including Gleason score, prostate-specific antigen (PSA) levels, stage and patient age ${ }^{1}$. While the

23 majority of patients with localized disease can be cured, some men recur with metastatic disease ${ }^{2}$

24 because of microscopic spread. The observed heterogeneity of outcomes might be explained by

25 heterogeneity within tumors ${ }^{3}$, which is missed by the current grading system and new prognostic

26 biomarkers are of utmost importance.

27 Several potential biomarkers including gene fusions, mutations, epigenetic heterogeneity,

28 and proteins have been studied ${ }^{4}$. Technological advances in proteomics now allow both 29 exploration of the proteome for biomarkers and assessment of the heterogeneity of biomarker 30 expression. However, analysis of a whole tissue core misses important cell-to-cell variability. In 31 ths study, we performed mass cytometry analysis of dissociated single cells from prostatectomies 
32 of 58 patients with tumors at varying grades and UICC (Union internationale contre le cancer)

33 stages using a set of 36 metal-tagged antibodies that recognize surface markers, enzymes,

34 transcription factors, and markers of functional readouts selected to facilitate characterization of

35 the phenotypic diversity of prostate tumors and their microenvironment. The power to

36 comprehensively analyze heterogeneity of tumors by simultaneously measuring dozens of

37 markers in hundreds of thousands to millions of cells makes mass cytometry the ideal tool to

38 characterize single-cell subpopulations present in prostate or other tumors including those rare

39 populations that cannot be detected with lower parametricity or lower throughput methods.

a

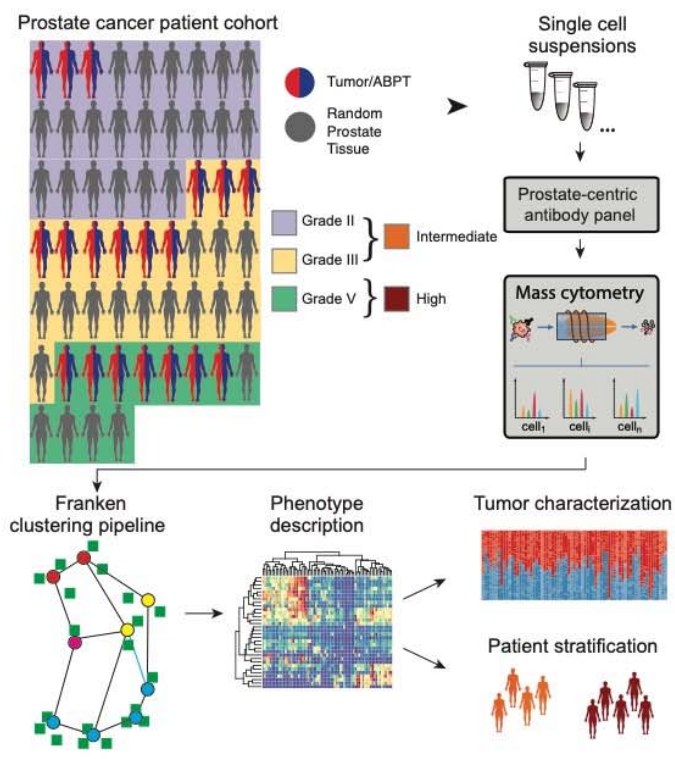

b

\begin{tabular}{|ll|}
\hline \multicolumn{1}{|c|}{ Antibody } & Description \\
\hline Immune context \\
\hline CD15 & Leukocytes \\
\hline CD20 & Granulocytes \\
\hline CD68 & B cells \\
\hline CD3 & Macrophages \\
\hline & T cells \\
\hline S100A4 & Fibroblasts \\
\hline Vimentin & Mesenchimally-derived \\
\hline SMA & Motility and invasion \\
\hline Ki-67 & Proliferation/Viability \\
\hline pH3 & Proliferation marker \\
\hline cl.PARP & Apoptosis marker \\
\hline cl.Caspase & Apoptosis marker \\
\hline AR & Transcription factor \\
\hline EZH2 & Prostate nuclear receptor \\
\hline NKX3.1 & Histone methylation \\
\hline GCR & Prostate tumor suppressor \\
\hline PR & Prostate nuclear receptor \\
\hline p53 & Tumor suppressor \\
\hline
\end{tabular}

\begin{tabular}{|l|l|}
\hline Antibody & Description \\
\hline & Epithelial cells \\
\hline EpCAM & Pan epithelial \\
panCK & Pan epithelial \\
E-Cadherin & Pan epithelial \\
\hline CK18 & Luminal cells \\
\hline CK8 & Luminal cells \\
\hline CK5 & Basal cells \\
\hline CK14 & Basal cells \\
\hline CK7 & Transitional epithelial cells \\
\hline CK19 & Transitional epithelial cells \\
\hline \multicolumn{3}{|c|}{ Prostate cells } \\
\hline PSA & Prostate epithelium \\
\hline Prostein & Prostate epithelium \\
\hline PSMA & Prostate epithelium \\
\hline AMACR & Prostate cancer \\
\hline & Other \\
\hline PTEN & Tumor Suppressor \\
\hline CA9 & Hypoxia \\
\hline CD31 & Endothelial cells \\
\hline CD24 & Stem cells \\
\hline Synaptophysin & Neuroendocrine cells \\
\hline
\end{tabular}

Figure 1. Schematic of method for characterization of primary prostate cancer tissue using mass cytometry. (a) The patient cohort consisted of 58 primary prostate cancer cases. For 16 patients, tumor and adjacent benign prostatic tissue (ABPT) samples were available. The remaining samples were from randomly selected regions from a prostatectomy without tumor assessment. Samples were analyzed by mass cytometry, and data were analyzed using Franken. (b) Markers used to categorize prostate epithelial cells as luminal, basal, or transitional, and markers used to identify tumor cells, cells from the microenvironment and functional features, such as proliferation, apoptosis or hypoxia.

Although mass cytometry has single-cell resolution capabilities, there are statistical challenges involved in analyzing such high-dimensional data. State-of-the-art clustering methods either underperform in precision and recall or require unnecessarily long runtimes and prohibitive computational resources ${ }^{5}$. To address this issue we developed an unsupervised,

44 single-cell clustering approach, Franken, which is unmatched in its combination of speed and 45 performance. Use of Franken to quantify the phenotypic diversity of single cells in prostate 46 tumor samples identified unique progression-related single-cell phenotypes. We detected 47 immune landscape features unique to patients with high grade prostate cancer, reflected by 
higher frequencies of macrophage and $\mathrm{T}$ cell phenotypes than observed in patients with intermediate grade disease. Further, we observed tumor-specific prostate epithelial phenotypes, including AR-negative and/or PSA-negative phenotypes typically associated with resistance to ADT and $\mathrm{CRPC}^{6-8}$, and previously undescribed and rare $\mathrm{CD} 15^{+}$phenotypes.

RESULTS

Clustering of high-dimensional mass cytometry data defines molecular profiles of prostate subpopulations

Using mass cytometry, we profiled tumor samples from 58 prostate cancer patients, including 24 patients with the International Society of Urological Pathology (ISUP) grade II (Gleason score 3+4), 22 grade III cases (Gleason score 4+3), and 12 patients with grade V prostate carcinomas (Gleason scores $4+5,5+4$ or $5+5$ ) (Figure 1a). Fresh tissue samples for dissociation into single cell suspensions were collected from 58 prostate cancer patients. From 41 of $58(71 \%)$ patients, the tumor could not be demarcated macroscopically. The presence of prostate cancer was confirmed histologically after examining the opposite side of the specimen, first in the frozen section and then after paraffin embedding; these samples are referred to as random prostate tissue (RPT) samples. For a further 17 patients (29\%), paired samples were taken from a macroscopically visible tumor mass and adjacent benign prostatic tissue (ABPT); tumor samples were validated using a frozen section of the opposite side. ABPT specimens were taken from the contralateral transitional zone of the prostate and never from the peripheral one, where tumor is more likely to be located. Single-cell suspensions from all prostate tissue samples and from 10 cell lines, including prostate cancer, stromal, and immune cells (Supplemental table 1), were barcoded, pooled and stained with a 36-antibody prostate cancer-centric panel, before mass cytometry acquisition. The antibody panel was designed to quantify markers that identify prostate epithelial cells, cells of the stroma and immune microenvironment, and markers of proliferation and survival (Figure 1b). Data for a total of 1,670,117 live cells were generated.

The high dimensionality of this dataset represented a challenge for data visualization and clustering. To address this task, we developed a highly efficient computational pipeline called Franken (Supplemental Figure 1a). The pipeline begins by building a large self-organizing map (SOM), which is used to fit all of the data. The SOM nodes in the original high-dimensional space are then used to build a mutual nearest neighbor graph using the Tanimoto similarity ${ }^{9}$ (also 
79 known as extended Jaccard similarity) that is subsequently clustered via the Walktrap graph

80 clustering technique ${ }^{10}$. Although all results presented in this paper were obtained in an

81 unsupervised manner, the pipeline also offers the option to define the chosen number of clusters

82 (detailed description found in methods). We demonstrated the F1 performance and scalability of

83 Franken when compared to state-of-the-art methods on two independent mid-size CyTOF

84 datasets (around 200,000 cells each; Supplemental Figure 1b-d). We also showed that our

85 pipeline is robust to its parameters' choice and scalable up to dozens of millions of cells in a

86 synthetic dataset (Supplemental Figure 1e,f).

87 Franken's scalability and ability to resolve rare metaclusters made it uniquely suitable to

88 explore our new prostate cancer patient dataset containing 1,670,117 cells. Analysis of the

89 prostate cancer dataset using Franken identified a total of 55 clusters (Figure 2a). Franken is a

90 very sensitive technique and leads to fine grained clusters that may represent the gradient

91 expression of certain markers without clear biological differences. It must be noted that discrete

92 labels do not easily apply to datasets with continuous expression such as single-cell data, where a

93 clear cutoff between cell states does not necessarily exist. Nonetheless, to obtain clusters that

94 were qualitatively different in terms of marker combination (which markers were expressed,

95 instead of how much), Franken clusters were further merged into 33 metaclusters using

96 hierarchical clustering of Pearson correlation dissimilarities with average linkage. We found 14

97 epithelial, 16 immune, one stromal and one endothelial phenotypes, based on marker expression

98 profiles (Supplemental figure 2a). We also identified one cluster which was mostly negative for

99 all 36 markers in the panel (denoted as NE01). Given that our panel does not cover the entire

100 proteome, this may represent a cell type not characterized by the markers in our panel or simply

101 outliers and was excluded from further analysis. All metaclusters were annotated using a two-

102 letter and two-digit identifier ranked by decreasing metacluster size (TC01 > TC02 > TC03 > ...)

103 for each cell category. The total number of cells in a cluster ranged from a few hundred (437

104 cells in EP01) to hundreds of thousands (391,554 in TC01; Supplemental Figure 2a).

105 In order to project the high dimensional data into a two-dimensional representation, we

106 used the UMAP (Uniform Manifold Approximation and Projection) method for dimensionality

107 reduction visualization ${ }^{29}$. UMAP showed that our analysis recapitulated the main cell-type

108 compartments within the prostate (Figure 2b); also detected were rarer cell states such as

109 apoptotic cells (TR02, AE01, AI01, MA03 and TC03). Franken was capable of resolving very 
110 rare populations present at frequencies as low as 1/5,000 (PR-high metacluster EP01; 111 Supplemental Figure 2b). FlowSOM, although very efficient in terms of speed, revealed fewer 112 and larger subpopulations than Franken; very rare populations identified by Franken could not be 113 identified by FlowSOM or PhenoGraph. Franken, therefore, provides an unprecedented 114 combination of performance, sensitivity and speed compared to existing clustering methods ${ }^{19}$. To

115 ensure good separability of each class (metacluster) and quality of the final clustering 116 configuration, we trained a logistic regression classifier with lasso regularization (using 5-fold 117 cross-validation to identify the regularization parameter $\lambda$; Supplemental Figure 2c,d). Most 118 metaclusters could be predicted with higher than $99 \%$ accuracy, and the lowest at $93 \%$.

119 All detected clusters contained cells from ABPT and tumor regions (Supplemental 120 Figure 3a). This suggested that tumor cells were present within the ABPT tissue and/or that 121 ABPT tissue was present inside the tumor mass which was expected due to the usual way 122 prostate tumors spread in the prostate as well as intrinsic limitations of the macroscopic-based 123 sample collection procedure (which could not ensure the adjacent regions were $100 \%$ tumor124 free). Alternatively, this could suggest our custom panel missed important markers to make such 125 distinction. 


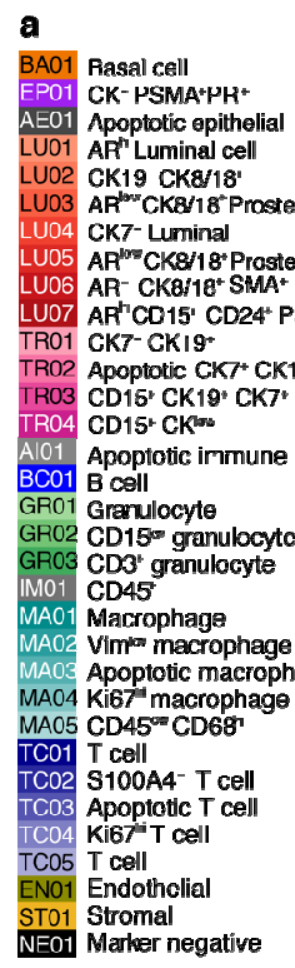

b

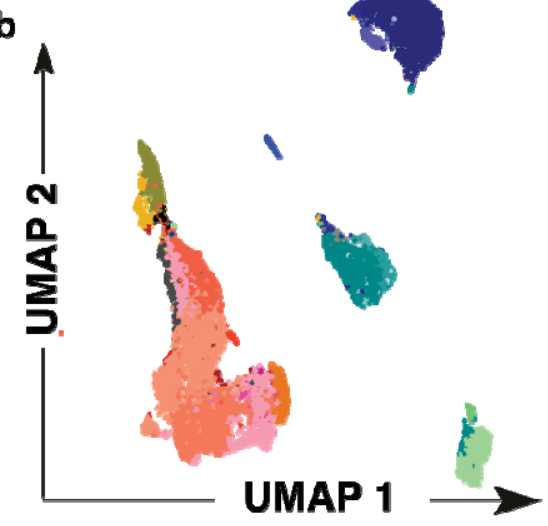

d

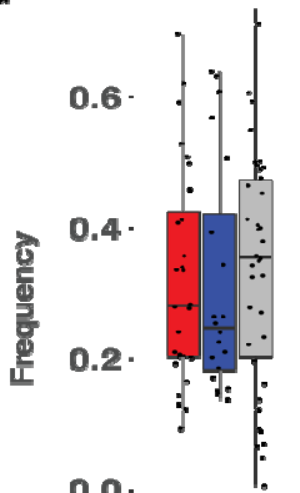

0.0

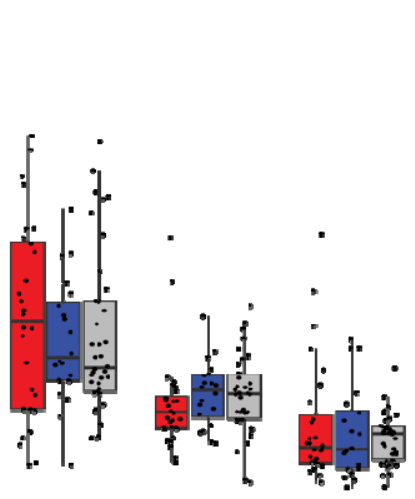

e

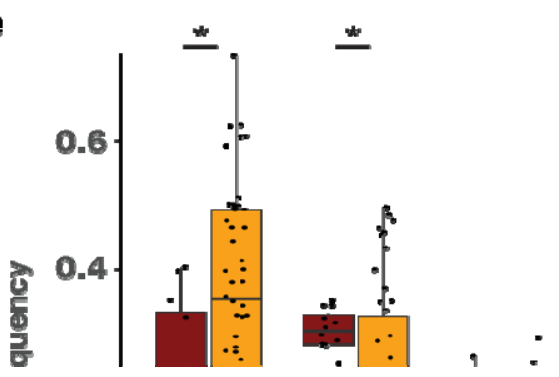

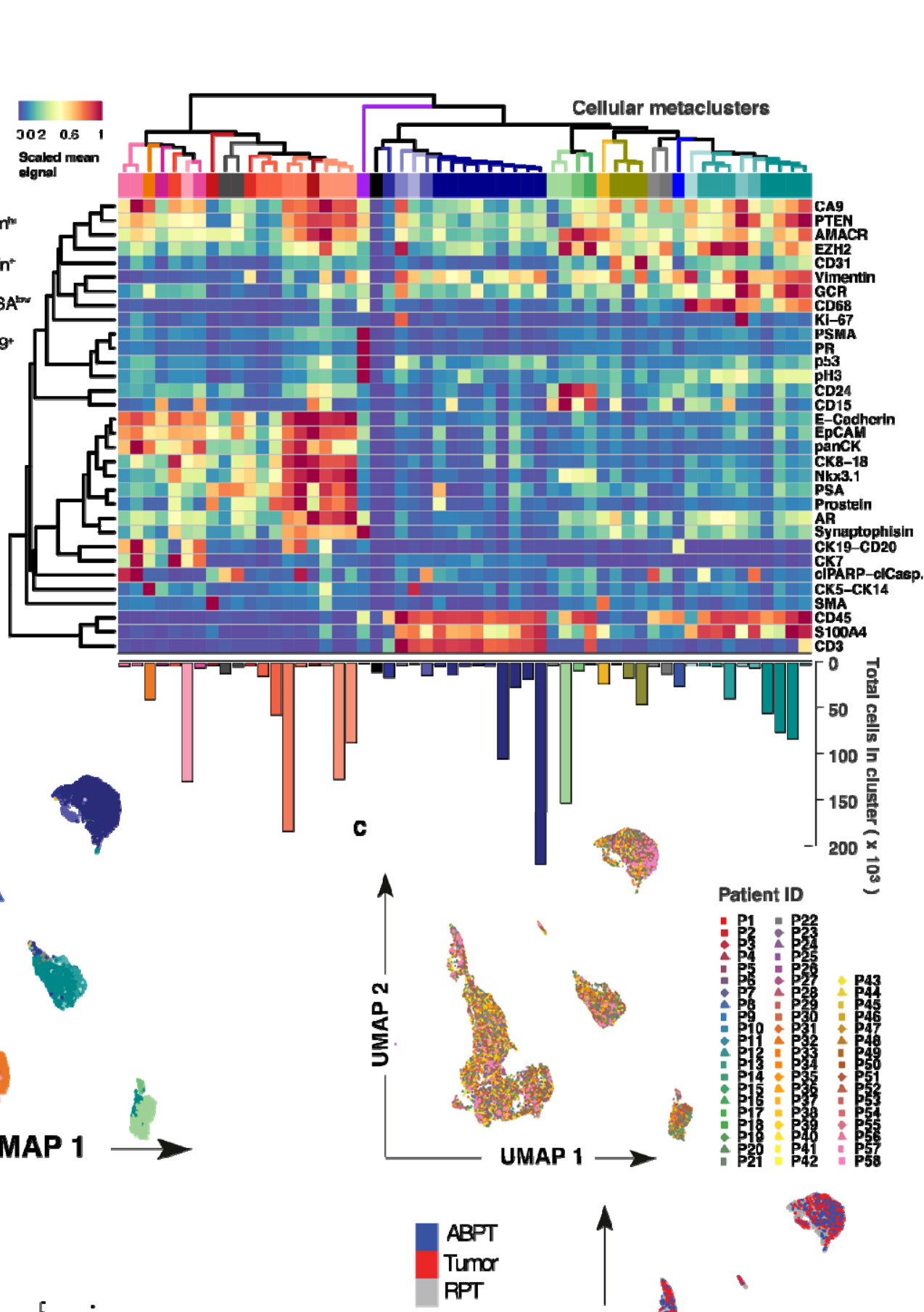

ABPT

Tumor

RPT
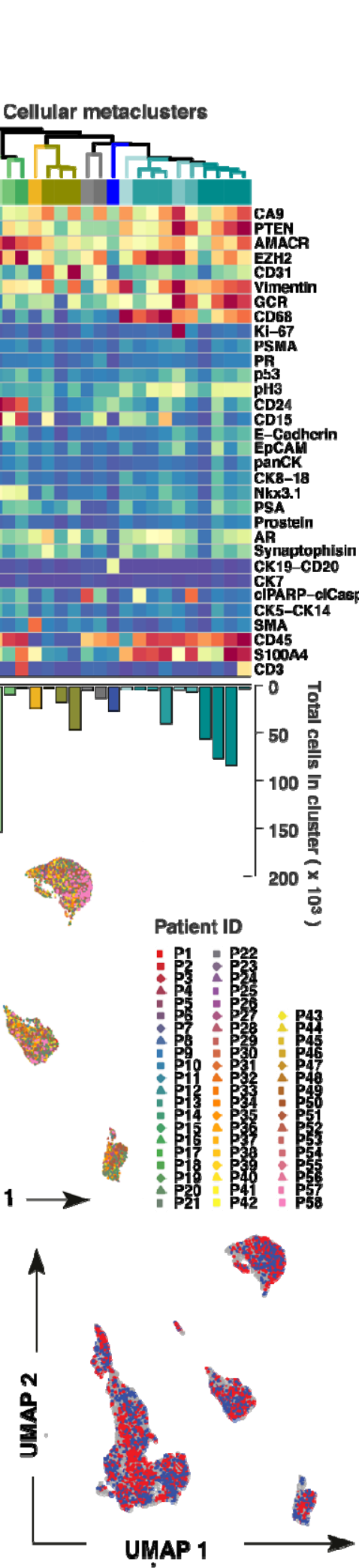

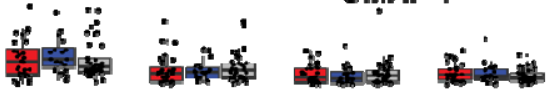


Figure 2. Prostate cancer samples have similar overlapping phenotypic profiles. (a) Heatmap of scaled mean signal of marker expression in 55 Franken clusters; numbers are colored according to metaclusters resulting from hierarchical clustering merging (using Pearson correlation dissimilarities) of Franken clusters. Bar plot below the heatmap corresponds to the number of cells found in each cluster. (b) UMAP map of 23,200 (400 per patient) cells colored by cellular metacluster as indicated in panel (a). (c) UMAP map of 23,200 (400 per patient) cells colored by patient. (d) (e) Boxplots of frequencies of the main cell types across all 58 samples from tumor, ABPT, and RPT. Significant changes were seen between tumor and ABPT in the proportion of granulocytes (two-sided Wilcoxon signed rank paired test, $\mathrm{p}=0.008$ ). $\mathrm{N}=17$. (e) Boxplots of frequencies of the main cell types in samples from all 58 patients in our cohort stratified by intermediate and high grade tumors. Changes in luminal and $\mathrm{T}$ cell compartments are significant according to a two-sided Mann-Whitney-Wilcoxon test ( $\mathrm{p}=0.028$ and 0.014 , respectively). Intermediate $\mathrm{N}=46$ and high grade $\mathrm{N}=12$.

Luminal cells were the most abundant cell type in the prostate and corresponded on 128 average (across all 58 patients) to $32 \%$ of a patient's sample; T cells were the second most 129 abundant population (24\% on average). When combined, cells from the immune compartment 130 and other cells of the tumor microenvironment made up over half the cells (54\% on average) 131 found within samples of this cohort (Supplemental Figure 3b). Franken clustering identified a 132 range of prostate epithelial phenotypes including a single basal cell phenotype characterized by 133 CK5 and CK14 expression (BA01), four transitional epithelial phenotypes expressing a 134 combination of CK7 and CK19 (TR01-04), and seven epithelial luminal phenotypes (LU01-07) 135 defined by the expressed CK8, CK18. Cellular metaclusters that contained a combination of CK7 136 or CK19 and CK8 or CK18 were annotated as luminal epithelial cells. Only CK7 and CK19137 positive metaclusters with very low to no CK8 and CK18 expression were denoted as transitional 138 epithelial cells. Luminal epithelial cells also expressed a combination (or varying expression 139 intensities) of AR, PSA, Prostein, Synaptophysin, AMACR, EZH2, PTEN and Nkx3.1. These 140 were weakly or not expressed in transitional or basal cell metaclusters.

141 In the microenvironment, five different $\mathrm{T}$ cell phenotypes were detected expressing CD3 142 and CD45, and five macrophage phenotypes were characterized by CD68 and CD45 expression. 143 Also detected were three granulocyte (expressing CD24 and/or CD15), one stromal 144 (characterized by SMA and S100A4), one B cell (CD20-expressing), and one endothelial (CD31145 expressing) clusters. Unlike in previous works ${ }^{11}$, we did not observe patient-specific batch 146 effects, which could have led to each patient clustering separately from one another. We found 147 that each metacluster contained a mixture of cells from most patients as illustrated in the UMAP 148 visualization (Figure 2c). 
There was considerable overlap in the single-cell phenotypes present within paired tumor and ABPT regions (UMAP; Figure 2d). Samples were stratified according to tumor, ABPT and RPT across cell types and we found significantly lower frequency of granulocytes in tumor regions than in ABPT (Figure 4e. Visualization of cells from intermediate grade (ISUP grades II and III) and high grade tumors (ISUP grade V) also revealed significant overlap (UMAP; Figure 2e) and indicated lower frequencies of luminal cells and higher frequencies of $\mathrm{T}$ cells in samples from patients with high grade compared to intermediate grade disease (Figure 2d).

\section{Immune landscape differs between tumor and benign adjacent tissue and across prostate cancer ISUP grade}

The UMAP visualization of 23,200 (400 per patient) cells, randomly selected across the patient cohort, revealed the expression patterns of markers associated with the microenvironment (Figure 3a and Supplemental Figure 3c). We validated both observations by quantifying $\mathrm{T}$ cells $\left(\mathrm{CD}^{+}\right)$and granulocytes $\left(\mathrm{CD} 15^{+}\right)$in a tissue microarray (Supplemental Figure $3 \mathrm{~d}$ and data availability section) that included formalin-fixed paraffin-embedded tissues from all patients in the cohort. Confirming the mass cytometry data, we observed higher densities of $\mathrm{T}$ cells in high grade tumors (Figure $3 \mathrm{~b}$ and $\mathrm{c}$ ) than in intermediate grade prostate tumors and lower densities of granulocytes in tumor regions than in ABPT regions (Figure $3 \mathrm{~d}$ and $3 \mathrm{e}$ ). 


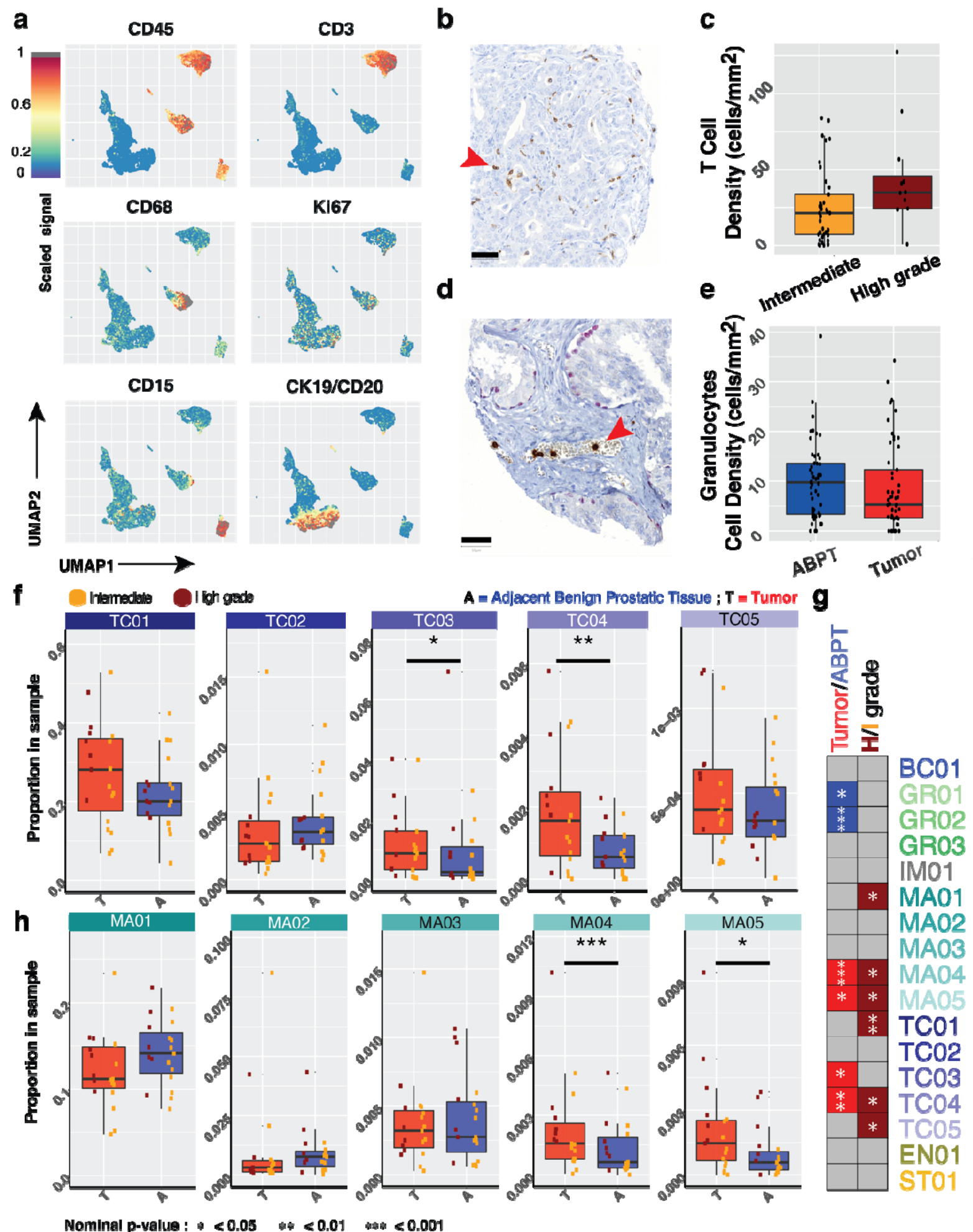

Figure 3. Stratification of samples reveals prostate tissue changes associated with tumor and advanced disease.

(a) UMAP of 23200 cells (400 per patient) colored by expression of indicated marker (b) Representative tissue sample stained for CD3 from a tissue microarray generated from prostate samples from the same cohort analyzed by mass cytometry. (c) Densities of T cells as determined by CD3 staining $(p=0.042)$. (d) Representative tissue sample stained for CD15 from a tissue microarray generated from prostate samples from the same cohort analyzed by mass cytometry. (e) Densities of granulocytes as determined by CD15 staining $(\mathrm{p}=0.058)$. Scale bar, $50 \mu \mathrm{m}$. (f) Proportion of T cell metaclusters in ABPT and tumor samples across patients 
with paired samples $(\mathrm{p}=0.066,0.169,0.023,0.002$ and 0.332 for TC01-05, respectively). N=17. (g) Summary table of clusters that were significant when comparing ABPT and Tumor samples ( $\mathrm{N}=17$ for both groups). Metaclusters enriched in ABPT are colored in blue while those enriched in tumor samples are colored in red. Comparison between intermediate and high grade patient samples (for combined tumor/ABPT; Intermediate $\mathrm{N}=46$ and high grade $\mathrm{N}=12$ ). Metaclusters enriched in patients with high grade disease are colored in dark red. Only significant relationships are colored and remaining comparisons are shown in grey. (h) Proportion of macrophage metaclusters in ABPT and tumor samples across patients with paired samples ( $\mathrm{p}=0.095$, $0.169,0.515,0.0004$ and 0.014 for MA01-05, respectively). $\mathrm{N}=17$. In panels (f) and (h) dots are colored by disease severity (intermediate vs high grade). In all boxplots, boxes illustrate the interquartile range (25th to 75 th percentile), the median is shown as the middle band, and the whiskers extend to 1.5 times the interquartile range from the top (or bottom) of the box to the furthest datum within that distance. Statistical testing between dependent paired Tumor and ABPT samples was done using a Wilcoxon signed rank paired-sample statistical tests (two-sided). Independent intermediate and high grade samples were tested using a twosided Wilcoxon rank sum test. Number of patients in each group is indicated by $\mathrm{N}$.

We identified multiple subpopulations of each immune cell type. By focusing on the comparison of each individual metacluster, we found that two $\mathrm{T}$ cell clusters were significantly enriched in tumor samples (TC03 and TC04, apoptotic T cells and proliferating $\mathrm{T}$ cells, respectively) when compared to the adjacent ABPT regions (Figure 3f). Notably, proliferating $\mathrm{T}$ cells were also enriched in high grade tumors and in high grade patient samples when tumor and adjacent tissue were mixed (Figure $3 \mathrm{~g}$ and Supplemental Figure 4a) suggesting that this $\mathrm{T}$ cell phenotype is enriched throughout the prostate of patients with high grade disease and not only in the core of the tumor.

The overall frequencies of macrophages were not significantly different between tumor and tumor-adjacent (ABPT) samples (Figure 2d). However, two macrophage metaclusters were enriched in tumor samples (MA04 and MA05, proliferating macrophages and CD45 ${ }^{\text {low }}$ macrophages respectively). These same metaclusters were further enriched in high grade patient samples (Supplemental Figure 4b). The overall macrophage proportion was actually lower in tumor samples than in tumor-adjacent samples, highlighting the importance of analyzing such a complex dataset at single-cell resolution to reveal that rare macrophages phenotypes can change in the opposite trend to the overall macrophage population. The majority of macrophages are localized in the prostate stroma, but their density is greater in tumorigenic regions ${ }^{12,13}$. This is a confounding factor when comparing macrophage frequencies across tumor grades, since lower grade tumors have a greater proportion of stroma than high grade tumors, resulting in a higher frequency of stroma-infiltrating macrophages (Figure 3h).

In summary, our clustering analysis identified changes in the cellular phenotypes present in the prostate tumor microenvironment compared to adjacent ABPT regions. Distinct macrophage phenotypes were associated with prostate tumors and with the stroma rich ABPT regions. Overall, the cell-type compositions of the tumor microenvironments differed with tumor 
grade, with the exception of granulocytes, which were decreased in tumor regions regardless of grade (Supplemental Figure 4c). Although the tumor microenvironment of the intermediate subcohort was characterized mostly by a relative decrease of immune phenotypes compared with ABPT regions, we found the opposite in the high grade sub-cohort where multiple immune phenotypes were enriched, notably highly proliferative macrophage and T cell phenotypes.

\section{Malignant and benign prostate tissues diverge in rare phenotypes}

Matched tumor and ABPT samples exhibited overall similar single-cell phenotypic profiles, and phenotypic profiles were similar across patients (Supplemental Figure 5b). These similarities were likely due to the presence of benign tissue in both tumor and ABPT samples, whereas patient-specific phenotypes are related to heterogeneous, deregulated malignant cells ${ }^{14}$.

206 We found that every sample from every patient, including tumor and ABPT samples, contained basal cells (BA01) as well as $\mathrm{CK}^{+} / \mathrm{CK} 19^{+}$live and apoptotic transitional epithelial cells (TR01 and TR02, respectively). All patient samples also contained a variety of luminal epithelial cells (LU01-LU07) containing varying combinations of luminal markers CK8/18, AR, PSA, Prostein, Nkx3.1 and in some cases the co-expression of CK19 and CK7 (LU02 and LU04, respectively).

212 04). Stem cell marker CD24 and neuroendocrine marker Synaptophysin showed highest 213 expression in luminal epithelial cells.

214 We carried out statistical comparisons for epithelial metaclusters and summarized the 215 significant relative enrichment results across all metaclusters (Figure 4a and Table S1) for 216 comparisons between patient grade groups (top row; $\mathrm{N}_{\mathrm{I}}=46, \mathrm{NH}_{H}=12$ ), tumor and ABPT 217 (middle row; $\mathrm{N}_{\mathrm{I}, \mathrm{H}}=17$ ), and high versus intermediate grade tumor regions only (bottom row; NI

$218=10, \mathrm{NH}_{\mathrm{H}}=7$ ). We identified the enrichment of apoptotic epithelial cells in tumor versus ABPT 219 regions, which was irrespective of tumor grade (Figure 4a and Supplemental Figure 5a). We also 220 found that luminal metaclusters were typically enriched in ABPT regions and/or in intermediate 221 stage patient samples. In particular, Prostein-high and AR-low metaclusters (LU03 and LU05; p 222 values $=0.0001$ and 0.026 respectively) were depleted in tumors versus ABPT samples (Figure 223 4a and Supplemental Figure 5c,d). The depletion of Prostein-high phenotypes was even more 224 pronounced in high grade compared to intermediate tumors (Figure 4a; bottom row). It is 225 possible that during tumor progression, regulation of differentiation programs is lost, and 
226 prostate-specific antigens are no longer expressed, supporting the hypothesis that aggressive 227 tumor cells are de-differentiated. A rare SMA-positive luminal cell type (LU06; p value $=0.012$ ) 228 was characteristic of patients with intermediate disease, irrespective of tumor or ABPT region. 229 The only luminal metacluster enriched in high grade patients was a rare PSA low, CD15+, $230 \mathrm{CD} 24+$ (an adhesion protein previously identified as a cancer stem-cell marker ${ }^{15}$ ) and AR-high 231 cell type (LU07; p value = 0.028; Figure 4a and Supplemental Figure 6). Two additional CD15+ 232 cell populations were identified, TR03 and TR04, amongst transitional epithelial metaclusters. 233 Both were increased in tumor and high grade patient samples, though significant enrichment 234 could only be detected in TR03 (p value $=0.003$ ) which co-expressed CK19 and CK7 (Figure 4a, 235 Supplemental Figure 5e,f) while TR04 may be a more common precursor with lower cytokeratin 236 expression. TR03 and TR04 also expressed a low amount of basal markers CK5/14. 
bioRxiv preprint doi: https://doi.org/10.1101/2021.01.25.428046; this version posted January 26, 2021. The copyright holder for this preprint (which was not certified by peer review) is the author/funder. All rights reserved. No reuse allowed without permission.

a

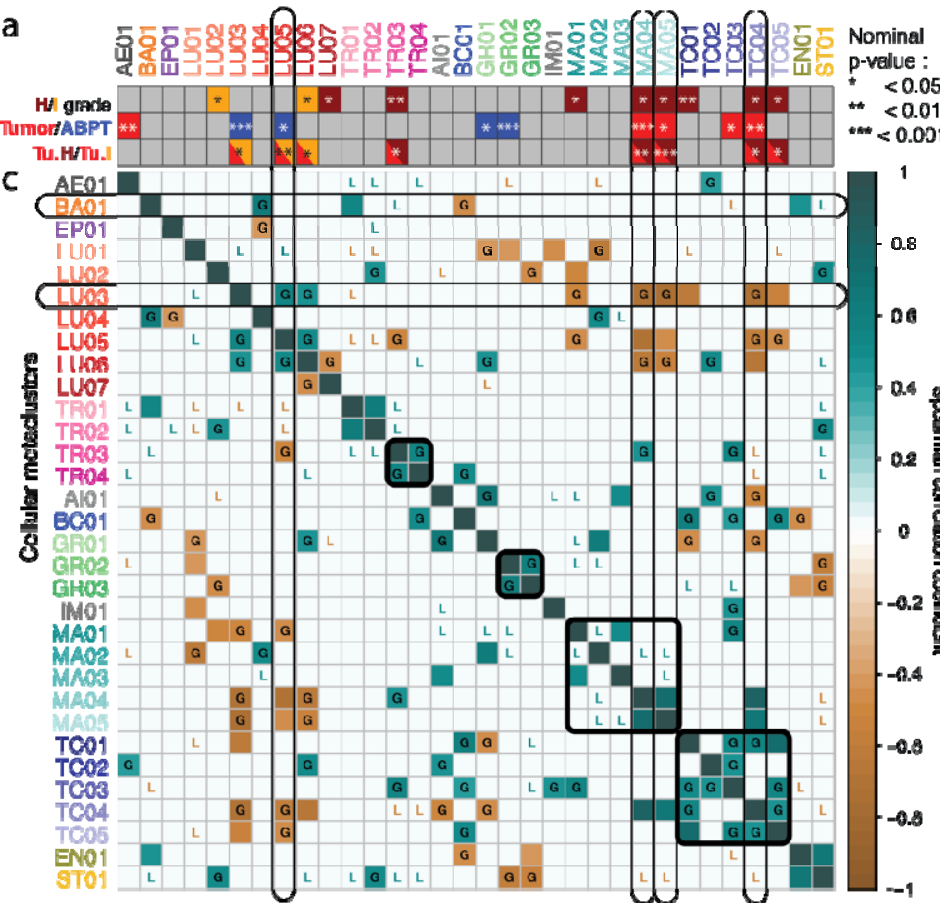

e

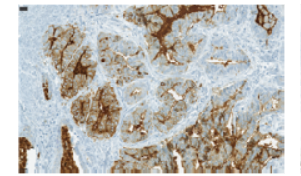

CD15 (brown) and pes (red)

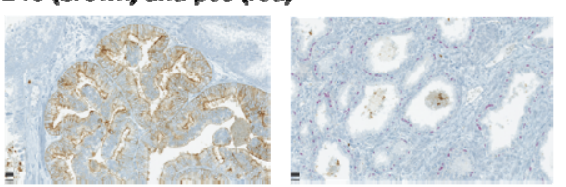

b

Epithellum Microenvi`onment

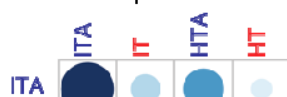

IT

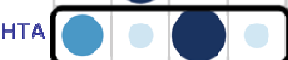

HT

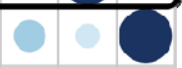

HT

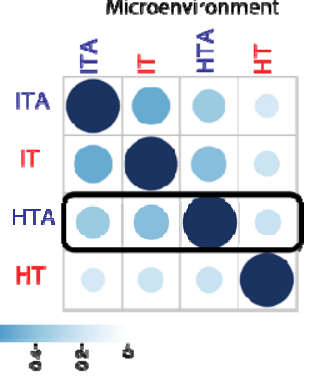

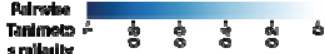

d
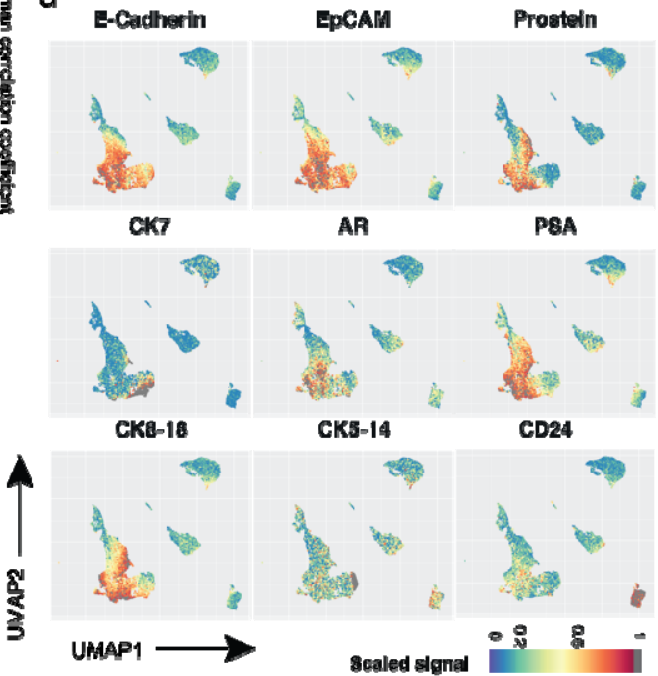

f

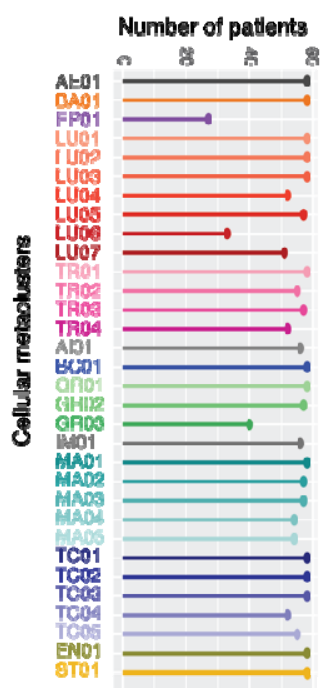

9
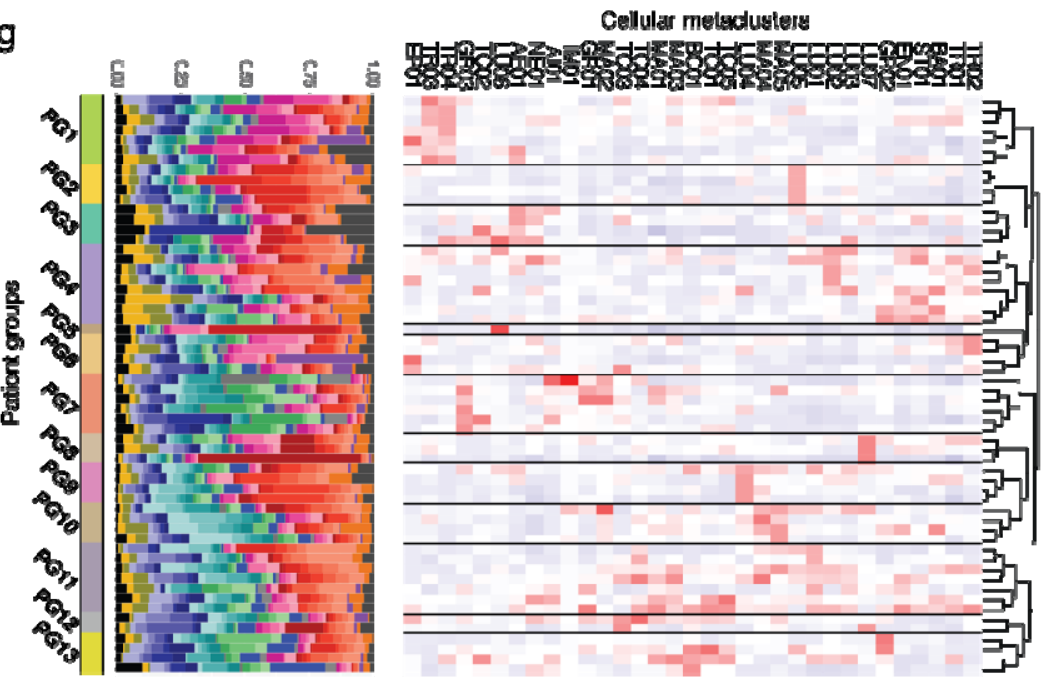

Figure 4. Characterization of epithelial tumor clusters and patient groups. (a) bar indicating which metaclusters were significantly enriched between three pairs of conditions: (top bar) high and intermediate grade samples, irrespective of tumor status; (middle bar) tumor versus ABPT; (bottom bar) intermediate and high grade tumor regions only. All nominal p values are given in Supplemental Table 2. (b) Pairwise Tanimoto similarity of intermediate (I) and high grade (H) tumor (T) and benign tumor-adjacent (TA) samples for metaclusters in the microenvironment and epithelium. (c) Correlation of metaclusters across 17 tumor patient samples. Correlations in the paired adjacent benign tissue that were lost in tumor are indicated by an L in the correlation plot while correlations that were gained are indicated by a G. Metacluster labels are colored to reflect cell types as in Figure 2a. (d) UMAP projections of 23200 cells ( 400 cells per patient) colored by expression of indicated epithelial and prostatespecific markers. Maximum signal $(=1)$ is shown in grey. (e) CD15 and p63 co-stained showing CD15 expression in both a patient with acinar (left) and another with ductal (middle) carcinoma as well as absence of CD15 in normal glands (right) 
showing basal cell layer expressing p63. Scale $25 \mu \mathrm{m}$. (f) Number of patients with cells belonging to a specific metacluster. Colors and labels matched to panels (a) and (c). (g) Grouped patient samples represented by proportion of metaclusters. Colors in bar plot reflect those on panels (a) and (c).

When interpreting changes in tumor and ABPT (Figure 4a, middle bar) in combination with tumor-only changes across grades (Figure 4a, bottom bar), we found that in some metaclusters (LU03, MA04, TC04) the effect between tumor and ABPT was stronger than the effect between patient grades. The depletion of LU03 and enrichment of MA04 and TC04 in tumors was observed across high and intermediate grade patients, however an effect could still

243 be observed between the different grade groups. In other cases (AE01, GR01, GR02 and TC03),

244 no effect could be detected between grades, only between tumor and ABPT samples, suggesting 245 that enrichment (AE01 and TC03; p values =0.003 and 0.022) or depletion (GR01 and GR02; p 246 values $=0.044$ and 0.0004$)$ of these cellular phenotypes happens in tumors of patients 247 irrespective of grade. Lastly, there were metaclusters that changed less significantly between 248 tumor and ABPT (LU05, TR03 and MA05; p values = 0.025, 0.096 and 0.014) and more across 249 grades ( $\mathrm{p}$ values $=0.004,0.026$, and 0.0007 ). This suggests that although there may have been a 250 difference between tumor and ABPT regions, a difference also existed between the tumor 251 regions of intermediate and high grade tumors. These cellular phenotypes suggest a possible 252 progressive change in the prostate, where some metaclusters are lowest in ABPT, higher in 253 intermediate grade tumors, and even higher in high grade tumors (or the reverse with highest 254 expression in ABPT and progressive loss in intermediate, then high grade tumors). We were also 255 interested in integrating information across all cellular phenotypes in the epithelium and in the 256 microenvironment (Figure 4b). We took the mean metacluster proportion across all 17 patients 257 for which we had tumor and ABPT (tumor adjacent) samples. We calculated the Tanimoto 258 similarity between intermediate tumor-adjacent regions (ITA), intermediate tumors (IT), high 259 grade tumor-adjacent regions (HTA) and high grade tumors (HT). We found that in the 260 epithelium, tumor-adjacent regions in intermediate and high grade are most similar to one 261 another, while intermediate and high grade tumors bear more similarity to each other than to 262 their benign adjacent regions. However, in the microenvironment, high grade tumor-adjacent 263 regions (HTA) are more similar to intermediate grade tumors (IT) than to their paired high grade 264 tumors (HT), suggesting that high grade tumors may have progressed from intermediate tumors, 265 but with further changes in epithelial cellular phenotypes, while the tumor-adjacent 266 microenvironment remains similar to that of an intermediate grade tumor (Figure 4b). 
Next, we analyzed correlations between metaclusters in tumor samples across the 17 patients for which we had paired tumor and ABPT samples (Figure 4c). We restricted the analysis to Spearman correlations with a significance level $<0.05$ for both correlations across tumor and ABPT samples. We compared correlations in the tumor to those in the paired adjacent

271 benign tissue and found that while some correlations were lost in tumor (indicated by an $\mathrm{L}$ in the 272 correlation plot; Figure 4c) others were gained (indicated by a G; Figure 4c). For example,

273 luminal epithelial cell types LU03 and LU05 were uncorrelated in benign tissue but correlated in

274 tumors. Indeed, we had found that both of these prostein-high metaclusters were depleted in 275 tumors and we found their decrease was correlated with one another. We found that while 276 transitional epithelial metaclusters TR01 and TR02 (CK7+/CK19+ cells and CK7+/CK19+ 277 apoptotic cells respectively) were correlated in both tumor and adjacent benign prostate tissue, 278 the strong correlation between TR03 and TR04 (CD15+/CK7+/CK19+ and CD15+/CK-low cells 279 respectively) was only present in tumor regions. New correlations also appeared between cell 280 types of the microenvironment. For example, GR02 and GR03 (CD15-low and CD3+ 281 granulocytes, respectively) were newly correlated in tumor regions. We also observed that most 282 T cell types became correlated in tumors although they were uncorrelated in adjacent benign 283 tissue. Anti-correlations were also gained. Notably, MA04, MA05 (proliferating and CD45-low 284 macrophages) and TC04 (proliferating T cells) became anti-correlated with LU03 in tumor. This 285 result suggests that the increase of these macrophage and $\mathrm{T}$ cell metaclusters may be related to 286 the depletion of this, likely benign, prostein-high luminal cell type.

287 While many new correlations and anti-correlations were gained in tumor regions, we also 288 found that previously (anti-)correlated cell types in benign tissue became uncorrelated in tumor 289 samples. Most macrophage metaclusters were correlated in benign tumor-adjacent samples but 290 no longer in tumor. Basal cells were correlated with TR03 but this was not the case in tumor 291 samples anymore, likely due to the de-regulated, malignant expansion of these transitional 292 epithelial cells. Apoptotic epithelial cells (AE01) were correlated with transitional metaclusters 293 TR01, TR02 and TR04 in tumor-adjacent regions but these correlations were lost in tumors. The 294 main transitional epithelial metacluster TR01 used to be anti-correlated with various, likely 295 benign, luminal cell types (LU01, LU03, LU05). It is believed that these transitional cells may 296 originate from basal cells and are precursors of luminal cells and therefore a balance between the 
population of $\mathrm{CK} 7+/ \mathrm{CK} 19+$ cells and CK8/18 exists in healthy scenario. However, our results support that this balance is disrupted in tumor regions.

The expression of CD15 in various malignant prostate epithelial cell populations (LU07, TR03 and TR04) was surprising although high-dimensional mapping using UMAP had already revealed CD15-high expressing cells in regions of epithelial marker expression such as ECadherin and EpCAM, luminal markers CK8 and CK18, and transitional epithelial markers CK7 and CK19 (Figures 3a and 4d). Immunohistochemistry confirmed the presence of CD15 in both ductal and acinar carcinoma of the prostate from patients who were shown to have this rare population by mass cytometry (one representative acinar and one ductal patient samples shown in Figure 4e). This adhesion molecule is typically used as a granulocyte marker and plays important roles in cell adhesion ${ }^{16}$ and migration ${ }^{17}$. Although previously observed in other carcinomas $^{18-20}$ and demonstrated to be a marker of propagating tumor cells ${ }^{21}$, cells expressing CD15 had not been previously detected in prostate tumors. To assess the clinical relevance of this CD15+/CK19+ subpopulation (metacluster TR03) we analysed two other TMA cohorts with 374 patients (example core shown in Supplemental Figure 7a) with localized disease (336) and

312 metastatic disease (38) and found that the proportion of patients with CD15 positive cells

313 increased with disease severity (low, intermediate and high ISUP grades) and was highest 314 amongst metastatic cases (Supplemental Figure 7b). Survival analysis did not yield any 315 differences for this cohort however, the number of CD15+/CK19+ cases was very low. Only 5\% 316 positive cases were detected (19 patients out of 374, for which survival data was only available 317 for 11) while via CyTOF most patients contained this cellular phenotype and 12\% (7 out of 58) 318 showed an enrichment. However, even in enriched cases, TR03 represented on average 0.6$3191.1 \%$ of cells in a patient and overall, across all patients, this cellular phenotypes represented on 320 average $0.3 \%$ of cells, making it difficult to identify a lot of potitive cases in a TMA spot with 321 diameter $0.06 \mathrm{~mm}$, here the number of cells is substantially lower than can be detected via high322 throughput mass cytometry. It is very likely many CD15-positive patients were missed in the 323 TMA analysis which impaired survival analysis. Overall, $0.1 \%, 0.3 \%$ and $0.1 \%$ cells were found across the whole dataset from LU07, 326 amongst the 14 observed in a subset of the patient cohort (Figure 4f). The remaining majority of metaclusters (29) were represented across all patients. In conclusion, our methodology identified 
328 a previously uncharacterized prostate-tumor subpopulation, which may also characterize a 329 distinct patient subtype.

330 Although elevated PSA levels are typically associated with localized prostate cancer,

331 luminal and transitional phenotypes found enriched in tumor or high grade samples had very 332 little or no PSA expression (AE01, TR03 and LU07). Prostate cancer cells that express low 333 levels or no PSA may be a self-renewing, tumor-propagating cell population that resists $\mathrm{ADT}^{7}$. 334 Similarly, not all phenotypes increased in tumor regions were high in AR. While AR 335 overexpression is associated with advanced disease and was found in one of the metaclusters 336 enriched in high grade tumors (LU07), an AR-low phenotype was also enriched in high grade 337 samples (TR03). Loss of $\mathrm{AR}$ has been associated with resistance to $\mathrm{ADT}^{8}$, and our results 338 support that malignant phenotypes are not necessarily high in AR. We also found that AR-low 339 metaclusters could be distinguished between benign and malignant by the presence of prostein 340 and PSA. AR-low phenotypes that were PSA- and prostein-high were benign (LU03 and LU05), 341 while malignant TR03, which was low in AR, was also low in PSA and prostein.

\section{Rare cellular phenotypes define patient subgroups.}

344 After having described the different cellular phenotypes present in prostate tumors, we wondered 345 whether certain metaclusters (or combinations) could characterize patient groups. We clustered 346 patients according to metacluster proportions using hierarchical clustering with Pearson 347 correlation dissimilarity. We found many small groups across our 58 patient cohort that could 348 each be characterized by the enrichment or depletion of a handful of phenotypes (Figure $4 \mathrm{~g}$ ). 349 After statistical testing, we were able to define which metaclusters significantly defined a patient 350 group (Figure 4g and Supplemental Figure 7c). Notably, patient group 1 consisted of patients 351 with enrichment of transitional epithelial cells expressing CD15 (TR03 and TR04). The former, 352 as had already been observed, was enriched in high grade tumors. Patient group 2 showed 353 significant enrichment of prostein high phenotype LU05. Group 3 consisted of patients with the 354 highest proportion of apoptotic epithelial cells (AE01), previously associated with tumor. One 355 patient, with the highest enrichment of SMA, metacluster LU06, clustered separately from the 356 rest and solely constituted patient group 5. High proportion of LU07 (CD15+, AR-high luminal 357 cells), which we had already shown as enriched in high grade tumors (Figure 4a), was 358 characteristic of patient group 8. The long-term effect that these phenotypes may have on 
survival remains to be determined. We showed that few single-cell phenotypes are necessary to further stratify patients beyond their ISUP tumor grade and may represent treatment targets for personalized treatment.

\section{DISCUSSION}

We achieved the first single-cell analysis of prostate tumors and tissues by mass cytometry using a newly developed computational method that provides an unprecedented combination of high-dimensional clustering performance and speed. To reveal the phenotypic diversity of primary prostate tumors and their microenvironment, we profiled 1,670,117 cells from 58 prostate cancer patients through simultaneous quantification of 36 different protein abundances using mass cytometry.

For data analysis, we used the newly developed Franken pipeline. The initial step builds a $\mathrm{SOM}^{22,23}$ to over-cluster the preprocessed data into a large number of nodes. Next, a mutual $k$ nearest neighbor graph is created between the SOM nodes using the Tanimoto similarity ${ }^{9}$. This

374 similarity measure is extremely suitable to analyzing high-dimensional data as it takes into 375 consideration both the angle and the length of vectors when indicating their proximity making it 376 more robust than more commonly used distance measures such as Euclidean or cosine. Lastly, 377 the resulting graph is clustered using a random-walk-based graph clustering technique called 378 Walktrap ${ }^{10}$. By comparing Franken to two state of the art methods for clustering mass cytometry 379 data we found that when compared to FlowSOM, Franken provided superior F1-scores. 380 Comparison of Franken to PhenoGraph showed that although they performed equivalently in F1381 scoring for data-sets of around 200,000 cells, Franken could be run on up to 40 million cells 382 (which would be computationally infeasible for PhenoGraph) in the time it would take 383 Phenograph to analyze 1 million cells. Franken also ran over 20 times faster than the state-of-the384 art single-cell RNA sequencing clustering technique, Seurat. Our Franken pipeline was able to 385 identify very rare subpopulations with a frequency as low as 1/5,000 and detected previously 386 unknown rare prostate cancer phenotypes which were later confirmed through imaging, showing 387 that Franken does not compromise performance or sensitivity while providing high scalability.

388 As Franken is sensitive, it can detect rare metaclusters based on subtle gradient variation 389 of markers. To focus on clusters with qualitatively similar expression patterns through 
hierarchical clustering of correlation similarities and described 33 prostate cellular phenotypes including 14 epithelial and 18 cell types from the microenvironment and one cluster with very low to no expression of the markers in our panel. Tumors and surrounding ABPT had considerable similarity: Nine of the 33 metaclusters (27\%) were present at significantly different

394 frequencies between the two regions. Although this was much higher than would be expected at random for a confidence level of 5\% (1.65 out of 33), almost two thirds or cell types and states detected (24 out of 33), were shared at similar proportions in tumor and ABPT tissue. Most epithelial differences between tumor and ABPT and tumor grades involved luminal cell types with the exception of one transitional epithelial phenotype (the CD15-high metacluster TR03), suggesting that prostate tumorigenesis is strongly affected by an interplay of luminal phenotypes. Prostein-high phenotypes were depleted in tumor regions and even more so in high grade tumors;

401 this suggests that during tumorigenesis there is selection for poorly differentiated cell types.

402 Tumor-enriched phenotypes all contained EpCAM. High levels of EpCAM expression at both 403 mRNA and protein levels were previously reported in prostate cancer tissues and cell lines ${ }^{24,25}$.

404 Our analysis showed that both AR-high /PSA-low (LU07) and AR-low /PSA-low (TR03) cells 405 were present in localized, hormone-naïve prostate tumors even though such phenotypes had 406 previously been associated only with castration-resistant disease after ADT or metastatic disease $407{ }^{6-8}$. Cells in the AR-high /PSA-low cluster also overexpressed Nkx3.1. Loss of prostein ${ }^{26}$ and 408 PSA expression ${ }^{27}, \mathrm{Nkx} 3.1$ overexpression $^{28}$, and AR overexpression or amplification ${ }^{29-31}$ have 409 been shown to be common in castration-resistant disease states. Furthermore, men with localized 410 high-grade prostate cancer but low PSA show inferior cancer survival ${ }^{32}$. It remains to be 411 determined whether these rare cells with the properties of tumors resistant to ADT are capable of 412 dissemination and are responsible for disease progression after prostatectomy. Surprisingly, two 413 phenotypes enriched in high grade patients expressed CD15. After analysing an additional 374 414 patients' TMA samples, we also found that CD15+/CK19+ prostate epithelial cells were further 415 enriched in metastatic disease. CD15 plays an important role in cell adhesion and migration ${ }^{16,17}$ 416 and CD15-expressing cells have been identified in other tumor types as having stem-like 417 potential but not in prostate cancer $^{18-20}$ and might represent a new biomarker for aggressive 418 phenotypes with a bigger potential to metastasize.

419 The tumor microenvironments were similar in both the tumor regions and the 420 neighboring ABPT regions for patients of different tumor grades with the exception of 
421 granulocytes, which were present at lower levels in tumors regardless of grade. Other rarer

422 immune cell types changed both between tumor and ABPT regions as well as across tumor 423 grades. In particular, we observed that one proliferating T-cell (TC04) and two macrophage

424 (proliferating MA04 and CD45-low MA05) phenotypes were enriched in tumor regions and were 425 further enriched in high-grade tumors.

426 The microenvironments of tumors from the intermediate sub-cohort had lower 427 frequencies of immune phenotypes compared to the ABPT regions, but we found the opposite in 428 the high grade sub-cohort. In high grade tumors, there was an enrichment of multiple immune 429 phenotypes compared to the ABPT regions. It is currently unclear whether intermediate grade 430 tumors progress to high grade disease. If such a progression happens, our analysis suggests that 431 the hyperplasia and expansion of the epithelial compartment might precede alterations in the 432 tumor microenvironment, or it may be that these differences are reflective of disease stage. We 433 proceeded to analyze the overall changes in the microenvironment and epithelium by integrating 434 information across all metaclusters in these two compartments and estimating the Tanimoto 435 similarity between high grade (HT) and intermediate tumors (IT) as well as high grade and 436 intermediate tumor-adjacent ABPT regions (HTA and ITA, respectively). We found further 437 evidence of a possible progression from intermediate to high grade tumors suggested by the 438 similarity of the microenvironments of intermediate grade tumors and high-grade ABPT regions. 439 Some of the rare cell types in the microenvironment, which we found to be enriched in tumors 440 from both grade groups and further enriched in high grade patients may represent new putative 441 targets that can be used to prevent the progression of the disease.

442 Tumors do not grow in isolation; cancerous cells require support from the 443 microenvironment. Accessory cells have been successfully targeted with therapy ${ }^{33-35}$. We found 444 that most immune metaclusters were present at similar frequencies or were decreased in the 445 tumor compared to the adjacent ABPT regions with the notable exceptions of rare $\mathrm{T}$ cell and 446 macrophages cellular phenotypes. Both monocyte infiltration and macrophage proliferation are 447 necessary for macrophage maintenance during tumor growth ${ }^{36}$, and in breast cancer, proliferating 448 macrophages are associated with high tumor grade, hormone receptor negativity, and poor 449 clinical outcome ${ }^{37}$. However, macrophage counting based on immunohistochemical analysis had 450 not led to any consensus on the prognostic significance of tumor-associated macrophages in 451 prostate cancer ${ }^{12,38}$. In our prostate cancer cohort, proliferative macrophages were enriched in 
452 prostate tumors and even more so in high grade patients. Taken together with findings that 453 tumor-associated macrophages (TAMs) are capable of proliferation ${ }^{37}$, our data suggests that in 454 addition to therapy that inhibits differentiation of TAMs from circulating monocytes, blocking 455 the proliferation of macrophages may have an effect in slowing the development of high grade 456 disease in prostate cancer. Our clustering analysis identified that not all macrophage phenotypes 457 changed frequency in tumor compared to ABPT regions. This suggests the presence of separate 458 cancer- versus stroma-infiltrating macrophage phenotypes that may have opposing influences on 459 tumorigenesis ${ }^{12,14}$, highlighting the importance of investigating macrophage infiltration in 460 prostate cancer.

461 In summary, new biomarkers are needed to identify which men qualify for active 462 surveillance or need aggressive treatment. Understanding the cellular complexity of prostate 463 tumors and their microenvironments is key to the development of new diagnostic and treatment 464 strategies. Here, we provide a thorough description of prostate tissue heterogeneity on the single465 cell level and describe differences between tumors and the neighboring benign hyperplasia 466 regions as well as across patient grades. We identify two CD15-high phenotypes enriched in high 467 grade patients as well as changes to the microenvironment in rare macrophage and $\mathrm{T}$ cell 468 phenotypes associated with tumor regions and high grade disease. We also identify in men with 469 localized disease, epithelial subpopulations associated with advanced castration-resistant disease.

470 The alterations to the epithelium and microenvironment should be further explored to guide 471 development of new diagnostic and treatment paradigms for prostate cancer and to understand 472 which cellular phenotypes in primary prostate cancer need to be detected and may change 473 treatment decisions.

\section{METHODS}

The Ethics Committee of the Canton of Zurich approved all procedures involving human prostate material (KEK-ZH-No. 2008-0040). All patients were part of the Zurich Prostate Cancer

480 Outcomes Cohort (ProCOC) study ${ }^{39,40}$, and each patient signed an informed consent form. 481 Prostatectomy samples were taken from 58 prostate cancer patients from the ProCOC cohort 482 between 2015 and 2017. Tumors were of a range of ISUP grades. No clinical or histological 
483 status was used in the selection of the cohort. Staging and grading was performed using World 484 Health Organization and ISUP criteria $^{41}$ ). Twenty-four patients had ISUP grade II (Gleason score $4853+4), 22$ had ISUP grade III (Gleason score 4+3), and 12 had ISUP grade V prostate carcinoma 486 (Gleason scores 4+5, 5+4, and 5+5).

487 Immediately after surgery, native radical prostatectomy specimens were transferred to the 488 frozen section lab on ice $\left(4^{\circ} \mathrm{C}\right)$ and were processed within $15 \mathrm{~min}$ in the Department of 489 Pathology and Molecular Pathology, University Hospital Zurich. The first slice after dissection 490 of the apex was quartered and snap frozen in four separate blocks for biobanking within the 491 ProCOC study. Fresh tumor and ABPT tissue was taken from the second slice after dissection of 492 the apex without destruction of surgical margins and the pseudocapsule. After formalin fixation 493 overnight, the rest of the specimen was embedded in paraffin. Hematoxylin and eosin stained 494 sections of the four frozen blocks were sliced for immediate evaluation regarding tumor load and 495 margins in synopsis with the standard formalin-fixed paraffin-embedded histology to control for 496 the representativeness of tissue sampling for mass cytometry.

497 Following evaluation of tissue sections by uropathologists (NJR, JHR, PJW) a tissue 498 microarray (TMA) containing two ABPT and two tumor regions from all patients in the selected 499 cohort was generated as previously described ${ }^{42}$. For TMA construction, representative tumor 500 areas of the second and third slice of radical prostatectomy specimens were chosen, as close as 501 possible to the area of tissue sampling for mass cytometry. Supplementary Figure 5 shows H\&E 502 images of the selected regions.

\section{$504 \quad$ Fresh tissue preparation}

505 After surgical resection and based on the aforementioned real-time frozen sections, the 506 index tumor lesions (the most extensive with the highest Gleason score) were immediately 507 harvested and transferred to precooled MACS tissue storage solution (Miltenyi Biotec) and 508 shipped at $4^{\circ} \mathrm{C}$. To better select the index lesion, only cases in which a tumor nodule was also 509 macroscopically visible were selected, ultimately resulting in a cohort with higher ISUP grades. 510 Tissue processing was completed within $24 \mathrm{~h}$ of collection. For the dissociation of tissues to 511 single cells, the tissue was minced using surgical scalpels and further disintegrated using the 512 Tumor Dissociation Kit, human (Miltenyi Biotech) and the gentleMACS Dissociator (Miltenyi 513 Biotech) according to the manufacturer's instructions. The resulting single-cell suspensions were 
514 filtered through sterile $70-\mu \mathrm{m}$ and $40-\mu \mathrm{m}$ cell strainers and stained for viability with $25 \mu \mathrm{M}$

515 cisplatin (Enzo Life Sciences) in a 1-min pulse before quenching with $10 \%$ FBS (Fienberg et al.,

516 2012). Cells were then fixed with 1.6\% paraformaldehyde (Electron Microscopy Sciences) for 10

517 min at room temperature and stored at $-80{ }^{\circ} \mathrm{C}$.

Mass cytometry barcoding

520 To ensure homogenous staining, $0.3 \times 10^{6}$ to $0.8 \times 10^{6}$ cells from each tumor sample were

521 barcoded as previously described using a 126-well barcoding scheme consisting of unique 522 combinations of four out of nine barcoding reagents ${ }^{43}$. Metals included palladium $\left({ }^{105} \mathrm{Pd},{ }^{106} \mathrm{Pd}\right.$, $523{ }^{108} \mathrm{Pd},{ }^{110} \mathrm{Pd}$, Fluidigm) conjugated to bromoacetamidobenzyl-EDTA (Dojindo) and indium $\left({ }^{113} \mathrm{In}\right.$ 524 and ${ }^{115}$ In, Fluidigm), yttrium $\left({ }^{89} \mathrm{Y}\right.$, Sigma Aldrich), rhodium $\left({ }^{103} \mathrm{Rh}\right.$, Sigma Aldrich), and bismuth 525 ( ${ }^{209} \mathrm{Bi}$, Sigma Aldrich) conjugated to maleimido-mono-amide-DOTA (Macrocyclics). The 526 concentrations were adjusted to $20 \mathrm{nM}\left({ }^{209} \mathrm{Bi}\right), 100 \mathrm{nM}\left({ }^{105-110} \mathrm{Pd},{ }^{115} \mathrm{In},{ }^{89} \mathrm{Y}\right), 200 \mathrm{nM}\left({ }^{113} \mathrm{In}\right)$, or 2 $527 \mu \mathrm{M}\left({ }^{103} \mathrm{Rh}\right)$ as previously reported to be optimal ${ }^{44}$. Cells were barcoded using the transient partial 528 permeabilization protocol ${ }^{45}$. Cells were washed with $0.03 \%$ saponin in PBS (Sigma Aldrich) and 529 incubated for $30 \mathrm{~min}$ at room temperature with $200 \mu \mathrm{l}$ of mass tag barcoding reagents. Cells were 530 then washed twice with PBS plus saponin and twice with cell staining medium (CSM, PBS with $5310.5 \%$ bovine serum albumin and $0.02 \%$ sodium azide).

\section{Antibodies and antibody labeling}

534 The supplier, clone, and metal tag for each antibody used in this study are listed in 535 Supplemental Table 3. Antibody labeling with the indicated metal tag was performed using the 536 MaxPAR antibody conjugation kit (Fluidigm). After metal conjugation, the concentration of 537 each antibody was assessed using a Nanodrop (Thermo Scientific). The concentration was 538 adjusted to $200 \mu \mathrm{g} / \mathrm{ml}$ and stored in Candor Antibody Stabilizer. All conjugated antibodies were 539 titrated for optimal concentration for use with prostate tissues. Antibody usage in this study was 540 managed using the AirLab cloud-based platform ${ }^{46}$.

\section{Immunohistochemistry}

543 For immunohistochemical validation studies anti-CD3 (mouse monoclonal, clone LN10, 544 Leica Microsystems) and anti-CD15 (mouse monoclonal, clone Carb-3, Agilent Dako) 
antibodies were used. Automated platforms were used for in situ protein expression analyses of CD15 (Ventana Benchmark CD15), and CD3 (Leica Bond-Max).

\section{Antibody staining and mass cytometry data collection}

After barcoding, pooled cells were incubated with FcR blocking reagent (Miltenyi Biotech) for $10 \mathrm{~min}$ at $4{ }^{\circ} \mathrm{C}$. Samples were stained with $100 \mu 1$ of the antibody panel per $10^{6}$ cells for $60 \mathrm{~min}$ at $4{ }^{\circ} \mathrm{C}$. Cells were washed twice in CSM and resuspended in $1 \mathrm{ml}$ of nucleic acid Ir-

552 Intercalator (Fluidigm) overnight at $4{ }^{\circ} \mathrm{C}$. Cells were then washed once in CSM, once in PBS,

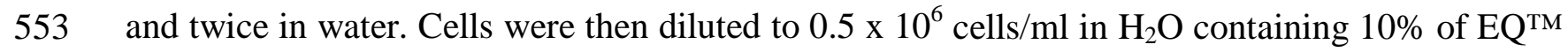

554 Four Element Calibration Beads (Fluidigm). Samples were placed on ice until analysis. Data 555 were acquired on an upgraded Helios CyTOF 2 mass cytometer using the Super Sampler 556 (Victorian Airship) introduction system.

\section{Mass cytometry data analysis}

Individual .fcs files collected from each set of samples were concatenated using the .fcs concatenation tool from Cytobank, and data were normalized using the executable MATLAB version of the Normalizer tool ${ }^{47}$. Individual samples were debarcoded using the CATALYST single-stained polystyrene beads as previously described ${ }^{48}$.

CyTOF data was analyzed by initially applying an arcsinh transformation with a cofactor

of 5 (newdata $_{i}=\operatorname{arcsinh}\left(\right.$ data $\left.\left._{i} / 5\right)\right)$. The UMAP algorithm ${ }^{49}$ was applied to the highdimensional data from 23,200 (400 per patient) cells taken at random from across the patient

567 cohort using default parameters (perplexity, 30; theta, 0.5) to facilitate visualization in two 568 dimensions. The pre-processed data were analyzed using the Franken algorithm as described in detail below. All analysis was done using R version 3.4.1

\section{The Franken pipeline}

The initial step of the Franken pipeline uses a SOM ${ }^{22,23}$ to over-cluster the preprocessed

573 data into a large number of nodes. Prostate patient data was pooled from all patient samples 574 (1,670,117 cells) and 400 SOM nodes were used (SOM grid dimensions were $\mathrm{x}=20$ and $\mathrm{y}=20)$.

575 Next, a mutual $k$-nearest neighbor graph $(\mathrm{k}=6)$ was built between the SOM nodes using the 
576 Tanimoto similarity ${ }^{9}$, which unlike the binary version, can be applied to continuous or discrete 577 non-negative features and retains the sparsity property of the cosine while allowing 578 discrimination of collinear vectors. Lastly, the resulting graph is clustered using a random-walk579 based graph clustering technique called Walktrap, using the implementations available in 580 package igraph ${ }^{50}$ in $\mathrm{R}$. Walktrap is a graph partitioning technique that requires a choice of 581 random walk steps. To increase our pipeline's robustness, this procedure is applied for a range 582 of random walk steps and the smallest step that maximizes the graph's modularity is chosen.

583 According to the thorough review and comparison of community detection algorithms by

584 Yang et al. ${ }^{51}$, Walktrap is amongst the best performing algorithms for both large and small 585 networks regardless of whether the mixing parameter is high or low. Although Yang et al. found 586 that for large mixing parameters most algorithms failed to detect the community structure, 587 Walktrap was able to do it. Another advantage of Walktrap is that it is possible (although not 588 necessary) for a user to define the number of communities one wishes to find in the data. This 589 allows the user to decide exactly how many clusters they wish to find, although the method is by 590 default run in an unsupervised way. Although Walktrap is not the fastest method for large 591 networks, the network size in Franken is never large due to the initial SOM-building step.

\section{Benchmarking Franken against other methods for additional datasets}

To test the performance of Franken, we compared it to two state-of-the-art clustering 595 methods for mass cytometry data, Phenograph ${ }^{52}$ and FlowSOM ${ }^{23}$. All three methods were used 596 to cluster data obtained from analysis of healthy bone marrow cells ${ }^{53}$ and data from 10 cell lines 597 stained with our prostate-centric antibody panel (Supplemental Figure 1b and 1c). The cell 598 phenotypes were manually annotated in the data obtained from analysis of the bone marrow cells 599 according to Bendall et al. ${ }^{53}$. We calculated precision and recall (as shown by F1 scores 600 according to Weber et al. ${ }^{5}$ ) for each phenotype in each dataset (Supplemental Figure 1a and 1b). 601 Franken was able to recover the most phenotypes in both datasets, resulting in the least 602 phenotypes with zero F1 scores. After repeating the F1 estimates for multiple runs (with different 603 random seeds) of each method, on average, Franken performed as well or better than the other 604 methods. Franken requires the input of three parameters: the SOM grid dimensions (x and y 605 which multiplied correspond to the number of nodes used to build the SOM) and k neighbors 606 (the number of neighbors used to decide whether two nodes are connected by an edge in the 
607 mutual nearest neighbor graph); a SOM size of $400(\mathrm{x}=\mathrm{y}=20)$ and $\mathrm{k}=6$ were used in our

608 simulations of all three datasets. Franken results were robust to the choice of its parameters 609 (Supplemental Figure 1).

$610 \quad$ Franken requires minimal computational resources, and runtimes were very fast when 611 evaluated on the two benchmark datasets containing around 200,000 cells (Supplemental Figure

612 2d). While FlowSOM was slightly faster than Franken, it performed very poorly in F1 scores 613 compared to both Franken and Phenograph. FlowSOM was run using the default parameters

614 chosen by the authors as optimal (SOM nodes 100). However, as FlowSOM and Franken share 615 the SOM building step we also tested FlowSOM using the same 400 nodes SOM grid however 616 the F1 scores were equivalent and the runtime was increased, no longer making FlwoSOM 617 superior in speed therefore we chose to use the author's default FlowSOM parameters. We 618 hypothesize that the poor performance of FlowSOM is due to the hierarchical clustering step 619 which is less suitable to the high dimensional node representation resulting from the SOM than 620 our mutual nearest neighbor graph-building approach.

Although the F1 scores from Phenograph were comparable to Franken's, their scalability

622 varied greatly. After testing Franken on several synthetic datasets of sizes varying from 20 623 thousand to 40 million, we showed that one could analyze 40 million cells with Franken in the 624 equivalent time taken to analyze 1 million cells using Phenograph (Supplemental Figure 2).

625 Franken can also be applied to single-cell RNA sequencing data, therefore we also compared 626 Franken's scalability with the state-of-the-art method for single-cell RNA sequencing Seurat and 627 could show that Franken was far superior in scalability (Supplemental Figure 2). Franken could 628 cluster 40 million cells in the half of the time taken to cluster 3 million cells with Seurat. As 629 Phenograph and Seurat require far larger computational resources they could not be run on the 630 larger datasets beyond 1 and 3 million respectively.

\section{Other computational methods}

633 PhenoGraph runs included in Supplemental Figure 1 were performed using the 634 MATLAB (R2018b) implementation using the GUI CYT3 as the matlab implementation was the 635 only one which allowed different random seeds to be used in each run. Default parameters were 636 used: $\mathrm{k}$ nearest neighbors $=30$. PhenoGraph runs in supplementary Figure 2 were performed 
637 using its implementation in $\mathrm{R}$ (Rphenograph) for its ease in including it in scripts instead of 638 manually running the MATLAB GUI.

639 FlowSOM and Seurat runs were performed using their implementation in $\mathrm{R}$ and default 640 parameters.

\section{TMA analysis}

642 Immunohistochemistry applied to TMA was used to validate single-cell mass cytometry 643 data. The open-source software QuPath ${ }^{54}$ was used to quantify cell types in TMA. CD3 ${ }^{+}$cells 644 were quantified using an automated detection procedure, and $\mathrm{CD}^{+}$cells were manually 645 selected by a pathologist (J.H.R.).

\section{Data availability}

648 The single-cell data supporting the findings of this study including raw .fcs files from primary 649 samples and cell lines as well as TMA images will be available online upon publication.

650 The Bone marrow CyTOF data pertaining to Figure 2 refers to article: 651 S.C. Bendall, E.F. Simonds, P. Qiu, A.D. Amir, P.O. Krutzik, R. Finck, R.V. Bruggner, R.Mela

652 med, A. Trejo, O.I. Ornatsky, et al. Single-cell mass cytometry of differential immune and drug 653 responses across a human hematopoietic continuum

654 Science, $332(2011)$, pp.687-696. The raw data is publicly available at

655 http://cytobank.org/nolanlab/reports

656 and was pre-processed according to Weber, Lukas M; Robinson, Mark D (2016). Comparison of 657 clustering methods for high-dimensional single-cell flow and mass cytometry data. Cytometry 658 Part A, 89(12):1084-1096.

\section{Code availability}

660 Code for data pre-processing pipeline (from Weber et al.) can be found here:

661 https://github.com/lmweber/cytometry-clustering-comparison

663 The Franken package is available at https://github.com/ldvroditi/Franken

\section{ACKNOWLEDGEMENTS}


667 The research was supported by a European Research Council PrECISE project from the 668 European Union Horizon 2020 research and innovation program under grant agreement No. 668858. We thank the Wild and Bodenmiller lab members as well as Dr. Andreas Moor for useful discussions and their valuable feedback.

\section{AUTHOR CONTRIBUTIONS} antibody panel and A.J. performed all antibody validation and data acquisition experiments. L.D.V.R. designed and developed Franken and performed data analysis. L.D.V.R and S.C tested algorithm in multiple datasets. T.H., C.P. and C.D.F. provided clinical samples. L.D.V.R. and P.W. developed TMA. P.W. P.B, J.H.R., A.T. and L.D.V.R. performed the TMA IHC image analysis. L.D.V.R. B.B. and P.W. performed biological analysis and interpretation with input from J.H.R and H.W.J.. L.D.V.R, B.B. and P.W. wrote the manuscript with input from F.C., H.W.J, S.C and C.D.F.

\section{COMPETING INTERESTS}

The authors declare no competing interests.

1. Dess, R. T. et al. Development and Validation of a Clinical Prognostic Stage Group System for Nonmetastatic Prostate Cancer Using Disease-Specific Mortality Results From the International Staging Collaboration for Cancer of the Prostate. JAMA Oncol. 6, 1912-1920 (2020).

2. Penney, K. L. et al. Gleason grade progression is uncommon. Cancer Res. 73, 5163-5168 (2013).

3. Guo, T. et al. Multi-region proteome analysis quantifies spatial heterogeneity of prostate tissue biomarkers. Life Sci. Alliance 1, (2018).

4. Cancer Genome Atlas Research Network. The molecular taxonomy of primary prostate cancer. Cell 163, 1011-1025 (2015).

5. Weber, L. M. \& Robinson, M. D. Comparison of clustering methods for high-dimensional single-cell flow and mass cytometry data. Cytometry A 89, 1084-1096 (2016).

6. Bluemn, E. G. et al. Androgen Receptor Pathway-Independent Prostate Cancer Is Sustained through FGF Signaling. Cancer Cell 32, 474-489.e6 (2017).

7. Qin, J. et al. The PSA(-/lo) prostate cancer cell population harbors self-renewing long-term 
tumor-propagating cells that resist castration. Cell Stem Cell 10, 556-569 (2012).

8. Li, Z. G. et al. Androgen receptor-negative human prostate cancer cells induce osteogenesis in mice through FGF9-mediated mechanisms. J. Clin. Invest. 118, 2697-2710 (2008).

9. Strehl, A. \& Ghosh, J. Value-based customer grouping from large retail data-sets. Data Mining and Knowledge Discovery: Theory, Tools, and Technology Ii 4057, 33-42 (2000).

10. Pons, P. \& Latapy, M. Computing communities in large networks using random walks. Computer and Information Sciences - Iscis 2005, Proceedings 3733, 284-293 (2005).

11. Jackson, H. W. et al. The single-cell pathology landscape of breast cancer. Nature 578, 615620 (2020).

12. Shimura, S. et al. Reduced infiltration of tumor-associated macrophages in human prostate cancer: association with cancer progression. Cancer Res. 60, 5857-5861 (2000).

13. Cao, J. et al. Prognostic role of tumour-associated macrophages and macrophage scavenger receptor 1 in prostate cancer: a systematic review and meta-analysis. Oncotarget 8, 8326183269 (2017).

14. Wagner, J. et al. A Single-Cell Atlas of the Tumor and Immune Ecosystem of Human Breast Cancer. Cell 177, 1330-1345.e18 (2019).

15. Ricardo, S. et al. Breast cancer stem cell markers CD44, CD24 and ALDH1: expression distribution within intrinsic molecular subtype. J. Clin. Pathol. 64, 937-946 (2011).

16. Forsyth, K. D., Simpson, A. C. \& Levinsky, R. J. CD15 antibodies increase neutrophil adhesion to endothelium by an LFA-1-dependent mechanism. Eur. J. Immunol. 19, 13311334 (1989).

17. Jassam, S. A. et al. TNF- $\alpha$ enhancement of CD62E mediates adhesion of non-small cell lung cancer cells to brain endothelium via CD15 in lung-brain metastasis. Neuro. Oncol. 18, 679690 (2016).

18. Kadota, A., Masutani, M., Takei, M. \& Horie, T. Evaluation of expression of CD15 and sCD15 in non-small cell lung cancer. Int. J. Oncol. 15, 1081-1089 (1999).

19. Jang, T. J., Park, J. B. \& Lee, J. I. The Expression of CD10 and CD15 Is Progressively Increased during Colorectal Cancer Development. Korean J Pathol 47, 340-347 (2013).

20. Brooks, S. A. \& Leathem, A. J. C. Expression of the Cd15 Antigen (Lewis-X) in BreastCancer. Histochemical Journal 27, 689-693 (1995).

21. Read, T.-A. et al. Identification of CD15 as a marker for tumor-propagating cells in a mouse model of medulloblastoma. Cancer Cell 15, 135-147 (2009).

22. Kohonen, T. The self-organizing map. Proc. IEEE 78, 1464-1480 (1990).

23. Van Gassen, S. et al. FlowSOM: Using self-organizing maps for visualization and interpretation of cytometry data. Cytometry A 87, 636-645 (2015).

24. Massoner, P. et al. EpCAM is overexpressed in local and metastatic prostate cancer, suppressed by chemotherapy and modulated by MET-associated miRNA-200c/205. Br. J. Cancer 111, 955-964 (2014).

25. Went, P. T. et al. Frequent EpCam protein expression in human carcinomas. Hum. Pathol. 35, 122-128 (2004).

26. $\mathrm{Xu}, \mathrm{J}$. et al. Identification and characterization of prostein, a novel prostate-specific protein. Cancer Res. 61, 1563-1568 (2001).

27. Wang, W. \& Epstein, J. I. Small cell carcinoma of the prostate. A morphologic and immunohistochemical study of 95 cases. Am. J. Surg. Pathol. 32, 65-71 (2008).

28. Xu, L. L. et al. Expression profile of an androgen regulated prostate specific homeobox gene NKX3.1 in primary prostate cancer. J. Urol. 163, 972-979 (2000). 
29. Bubendorf, L. et al. Survey of gene amplifications during prostate cancer progression by high-throughout fluorescence in situ hybridization on tissue microarrays. Cancer Res. 59, 803-806 (1999).

30. Koivisto, P. et al. Androgen receptor gene amplification: a possible molecular mechanism for androgen deprivation therapy failure in prostate cancer. Cancer Res. 57, 314-319 (1997).

31. Watson, P. A., Arora, V. K. \& Sawyers, C. L. Emerging mechanisms of resistance to androgen receptor inhibitors in prostate cancer. Nat. Rev. Cancer 15, 701-711 (2015).

32. Fankhauser, C. D. et al. Inferior Cancer Survival for Men with Localized High-grade Prostate Cancer but Low Prostate-specific Antigen. Eur. Urol. 78, 637-639 (2020).

33. Mills, C. D., Lenz, L. L. \& Harris, R. A. A Breakthrough: Macrophage-Directed Cancer Immunotherapy. Cancer Res. 76, 513-516 (2016).

34. Restifo, N. P., Dudley, M. E. \& Rosenberg, S. A. Adoptive immunotherapy for cancer: harnessing the T cell response. Nat. Rev. Immunol. 12, 269-281 (2012).

35. Mariathasan, S. et al. TGF $\beta$ attenuates tumour response to PD-L1 blockade by contributing to exclusion of T cells. Nature 554, 544-548 (2018).

36. Franklin, R. A. et al. The cellular and molecular origin of tumor-associated macrophages. Science 344, 921-925 (2014).

37. Campbell, M. J. et al. Proliferating macrophages associated with high grade, hormone receptor negative breast cancer and poor clinical outcome. Breast Cancer Res. Treat. 128, 703-711 (2011).

38. Nonomura, N. et al. Infiltration of tumour-associated macrophages in prostate biopsy specimens is predictive of disease progression after hormonal therapy for prostate cancer. BJU Int. 107, 1918-1922 (2011).

39. Umbehr, M. et al. ProCOC: the prostate cancer outcomes cohort study. BMC Urol 8, 9 (2008).

40. Wettstein, M. S. et al. Prognostic role of preoperative serum lipid levels in patients undergoing radical prostatectomy for clinically localized prostate cancer. Prostate 77, 549556 (2017).

41. Epstein, J. I. et al. The 2014 international society of urological pathology (ISUP) consensus conference on gleason grading of prostatic carcinoma: definition of grading patterns and proposal for a new grading system. Am. J. Surg. Pathol. 40, 244-252 (2016).

42. Mortezavi, A. et al. KPNA2 expression is an independent adverse predictor of biochemical recurrence after radical prostatectomy. Clin. Cancer Res. 17, 1111-1121 (2011).

43. Zunder, E. R., Lujan, E., Goltsev, Y., Wernig, M. \& Nolan, G. P. A continuous molecular roadmap to iPSC reprogramming through progression analysis of single-cell mass cytometry. Cell Stem Cell 16, 323-337 (2015).

44. Zivanovic, N., Jacobs, A. \& Bodenmiller, B. A practical guide to multiplexed mass cytometry. Curr. Top. Microbiol. Immunol. 377, 95-109 (2014).

45. Behbehani, G. K. et al. Transient partial permeabilization with saponin enables cellular barcoding prior to surface marker staining. Cytometry A 85, 1011-1019 (2014).

46. Catena, R., Özcan, A., Jacobs, A., Chevrier, S. \& Bodenmiller, B. AirLab: a cloud-based platform to manage and share antibody-based single-cell research. Genome Biol. 17, 142 (2016).

47. Finck, R. et al. Normalization of mass cytometry data with bead standards. Cytometry A 83, 483-494 (2013).

48. Chevrier, S. et al. Compensation of signal spillover in suspension and imaging mass 

cytometry. Cell Syst. 6, 612-620.e5 (2018). (2008).

50. Gabor Csardi and Tamas Nepusz. The igraph software package for complex network research. InterJournal Complex Systems, 1695 (2006).

51. Yang, Z., Algesheimer, R. \& Tessone, C. J. A comparative analysis of community detection algorithms on artificial networks. Sci. Rep. 6, 30750 (2016).

52. Levine, J. H. et al. Data-Driven Phenotypic Dissection of AML Reveals Progenitor-like Cells that Correlate with Prognosis. Cell 162, 184-197 (2015).

53. Bendall, S. C. et al. Single-cell mass cytometry of differential immune and drug responses across a human hematopoietic continuum. Science 332, 687-696 (2011).

54. Bankhead, P. et al. QuPath: Open source software for digital pathology image analysis. Sci. Rep. 7, 16878 (2017).

808 


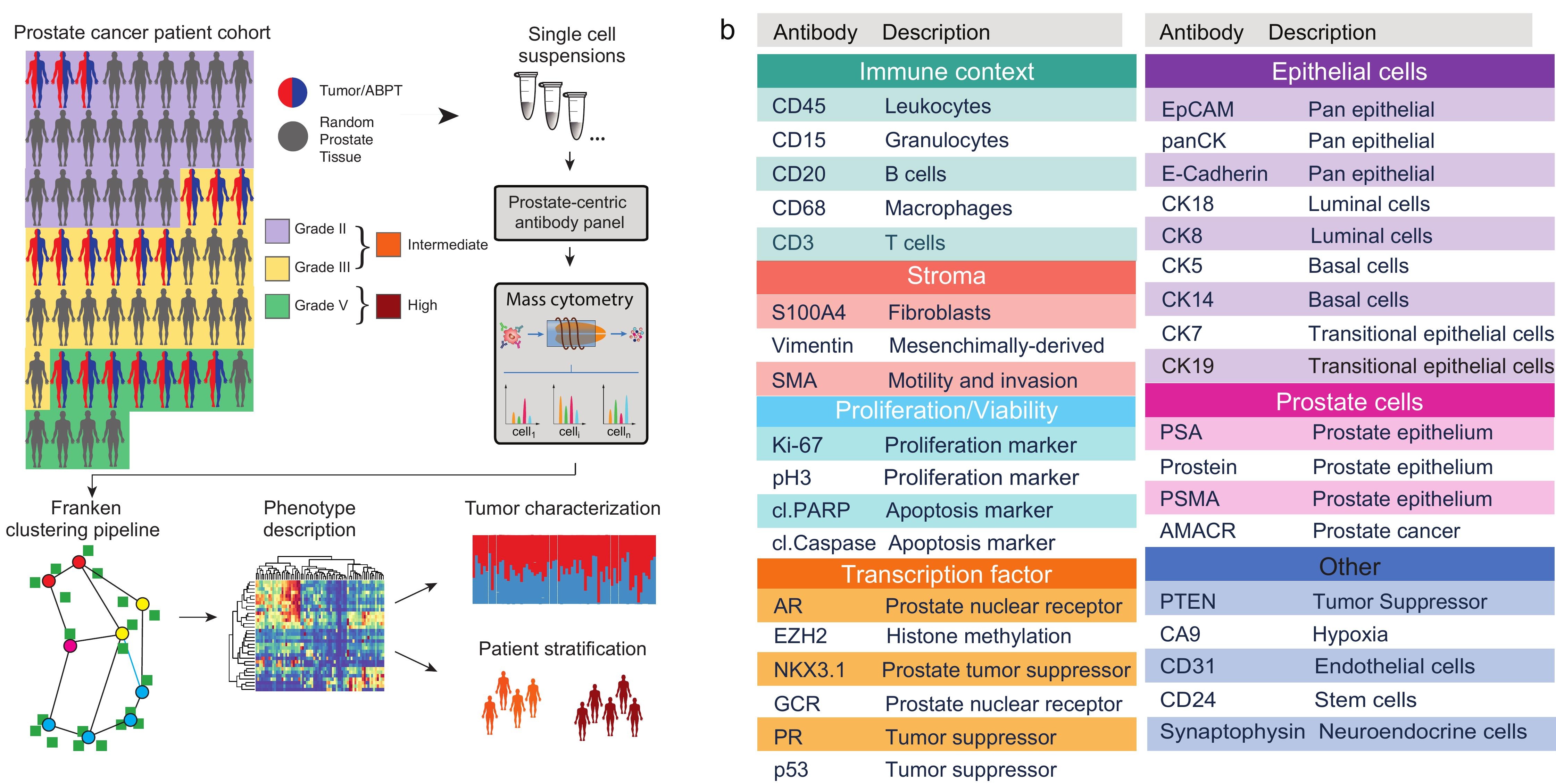



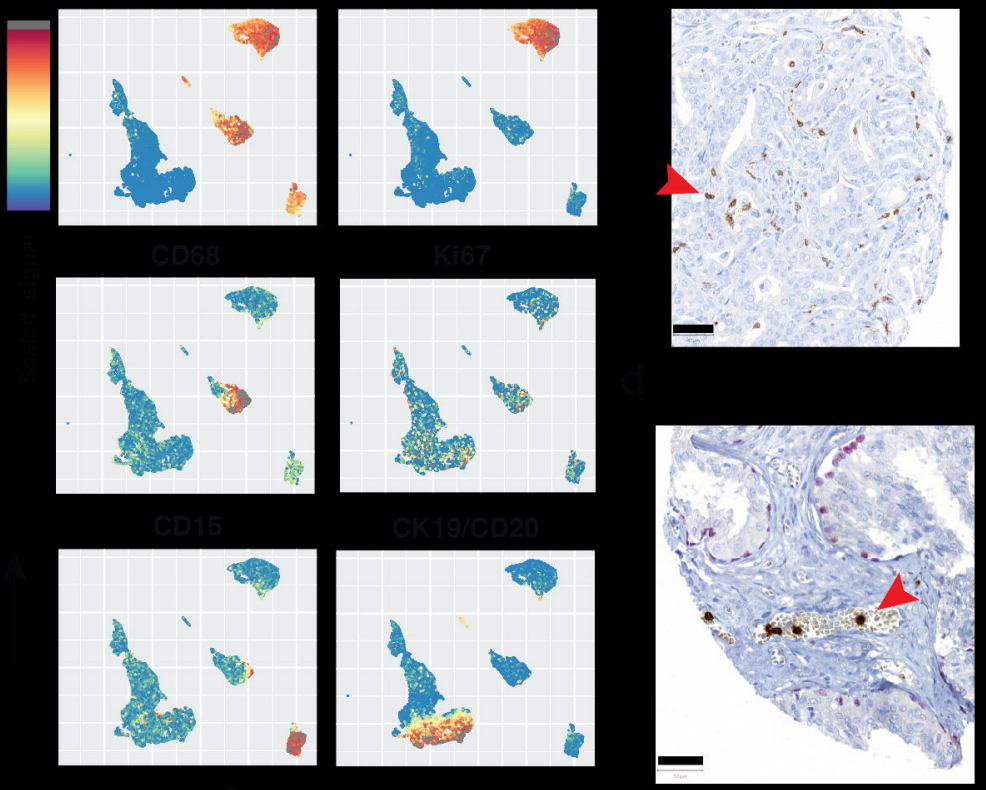

$=$ Adjacent Benign Prostatic Tissue = Tumor
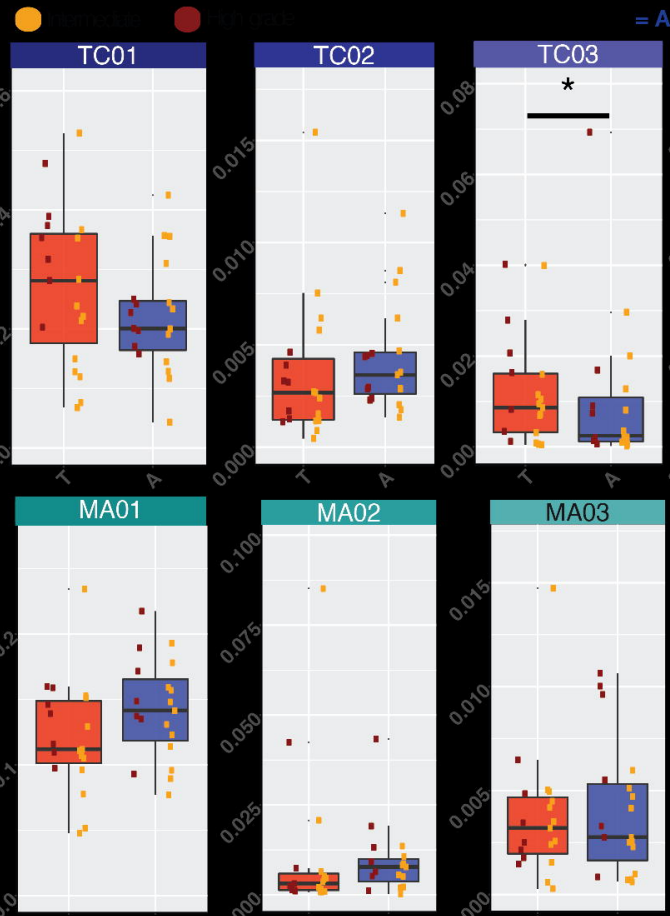
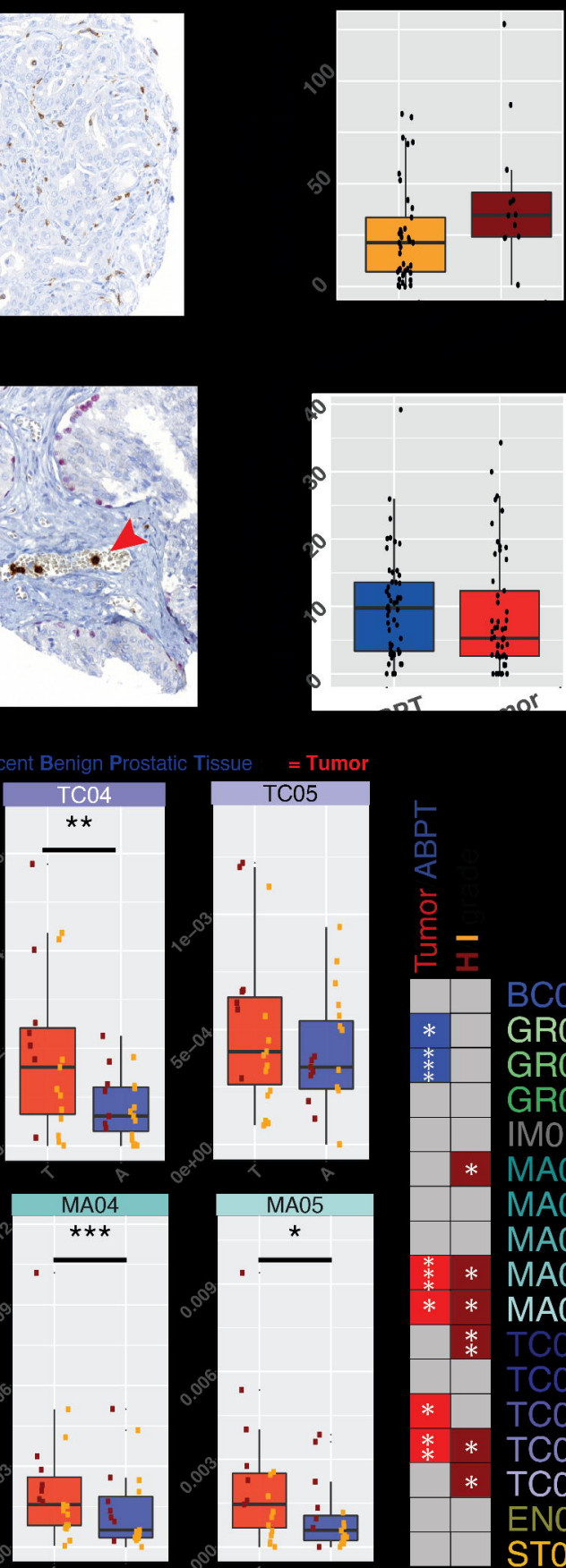

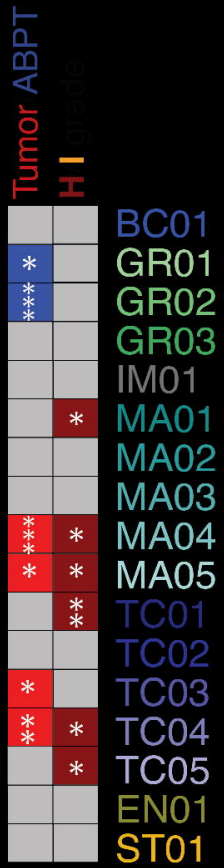




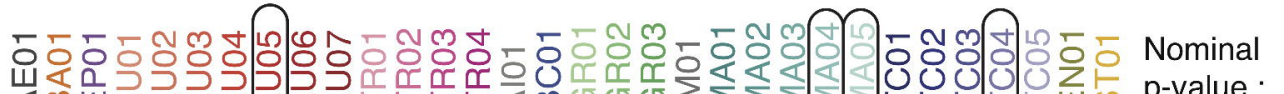

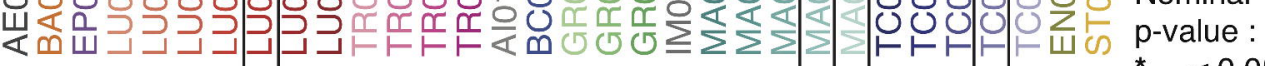

b

Epithelium

Microenvironment

**** $* * * D^{*}<0.05$ $\stackrel{\Xi}{上}$ 乒壬

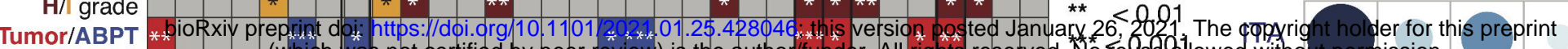
(Which was not certified by peer review) is the authorffunder. All rights reserved. Nô relsegillowed witho

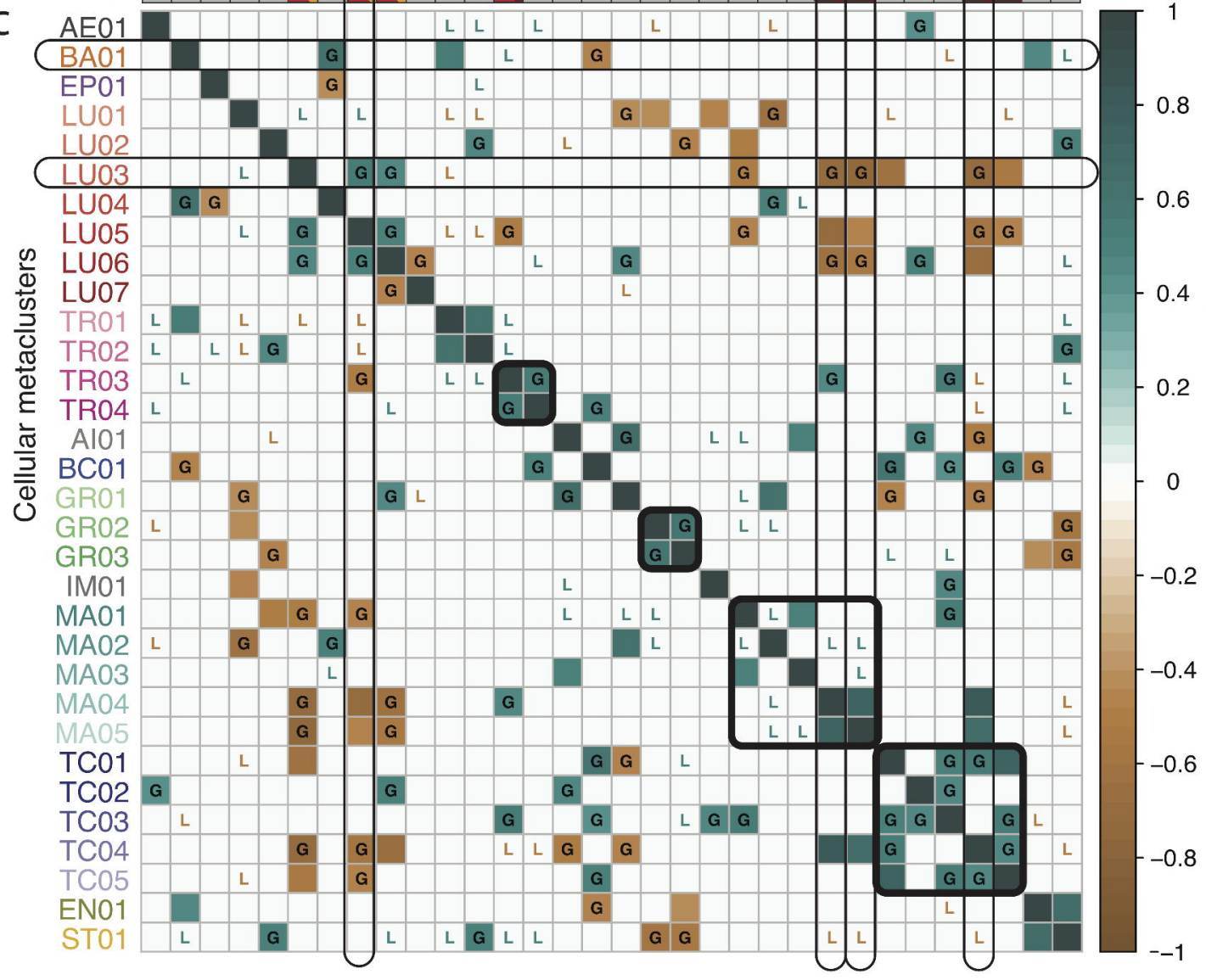

e

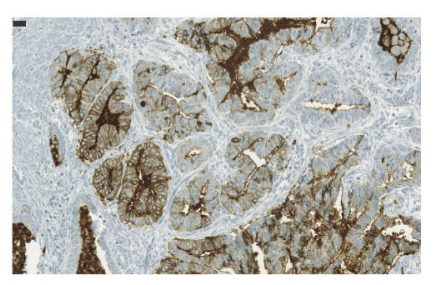

CD15 (brown) and p63 (red)

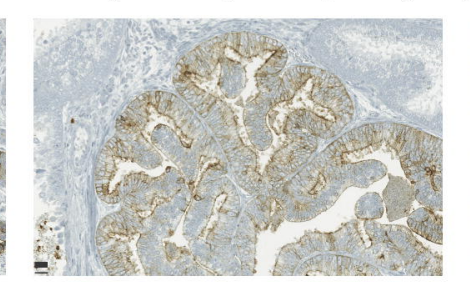

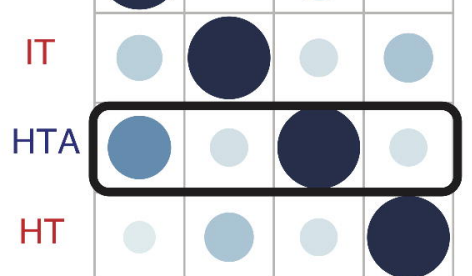

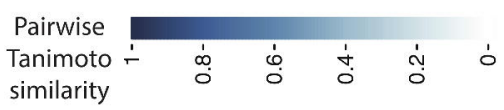

d

E-Cadherin

ㄷ․
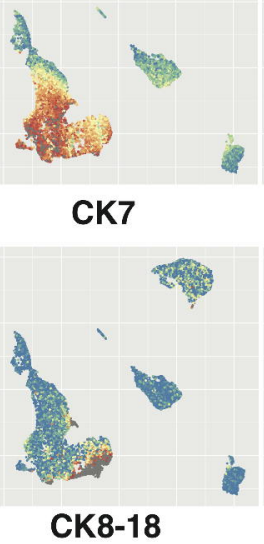

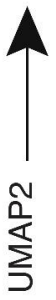

Number of patients $\quad \mathrm{g}$

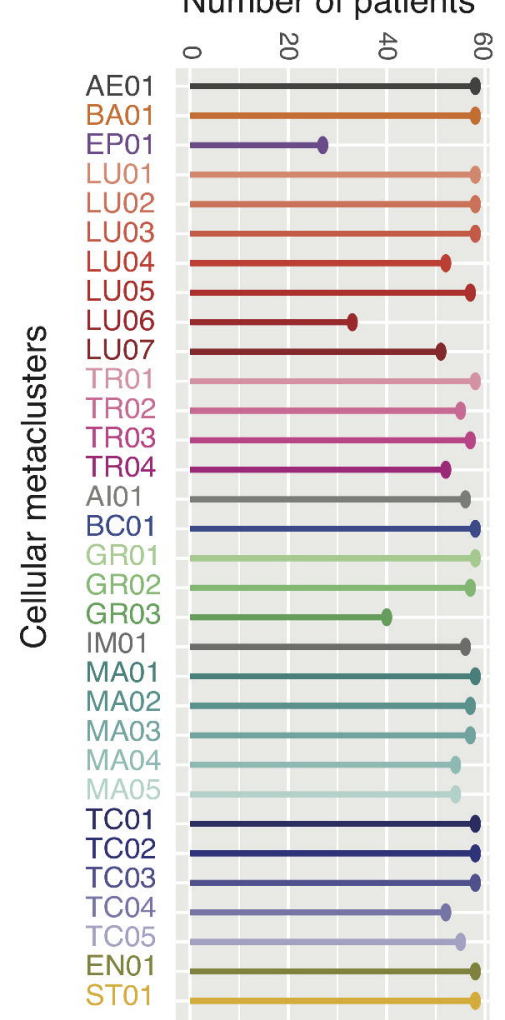

9

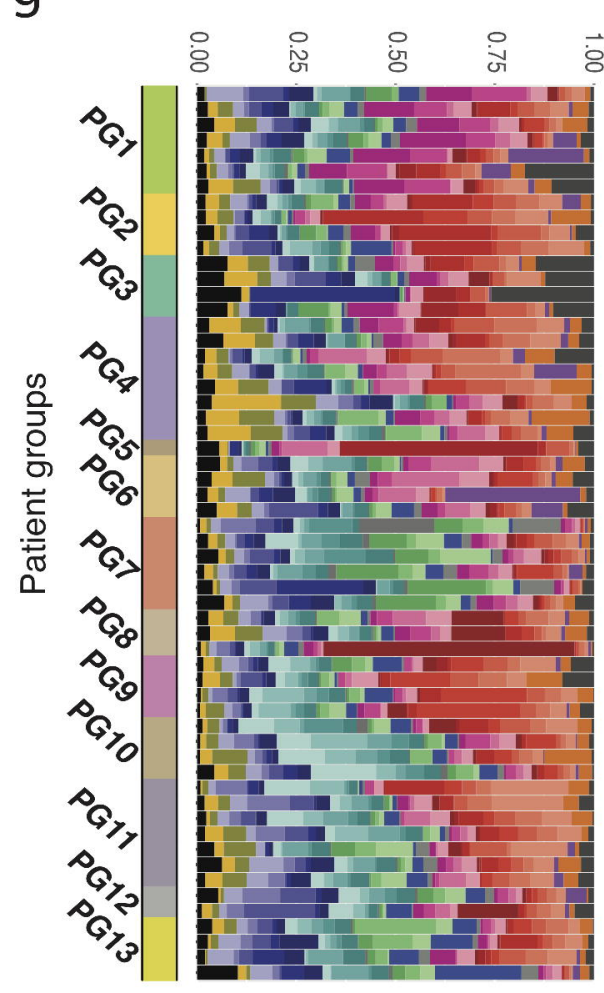

EPCAM

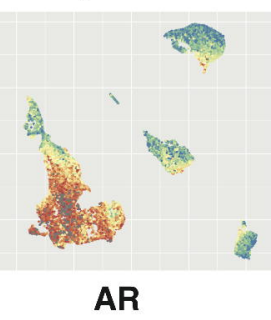

\$.

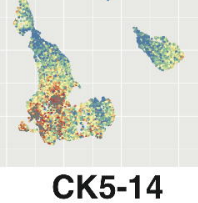

औ?

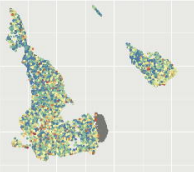

Scaled signal
Prostein
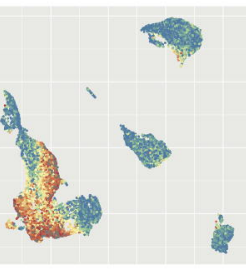

PSA
Cellular metaclusters

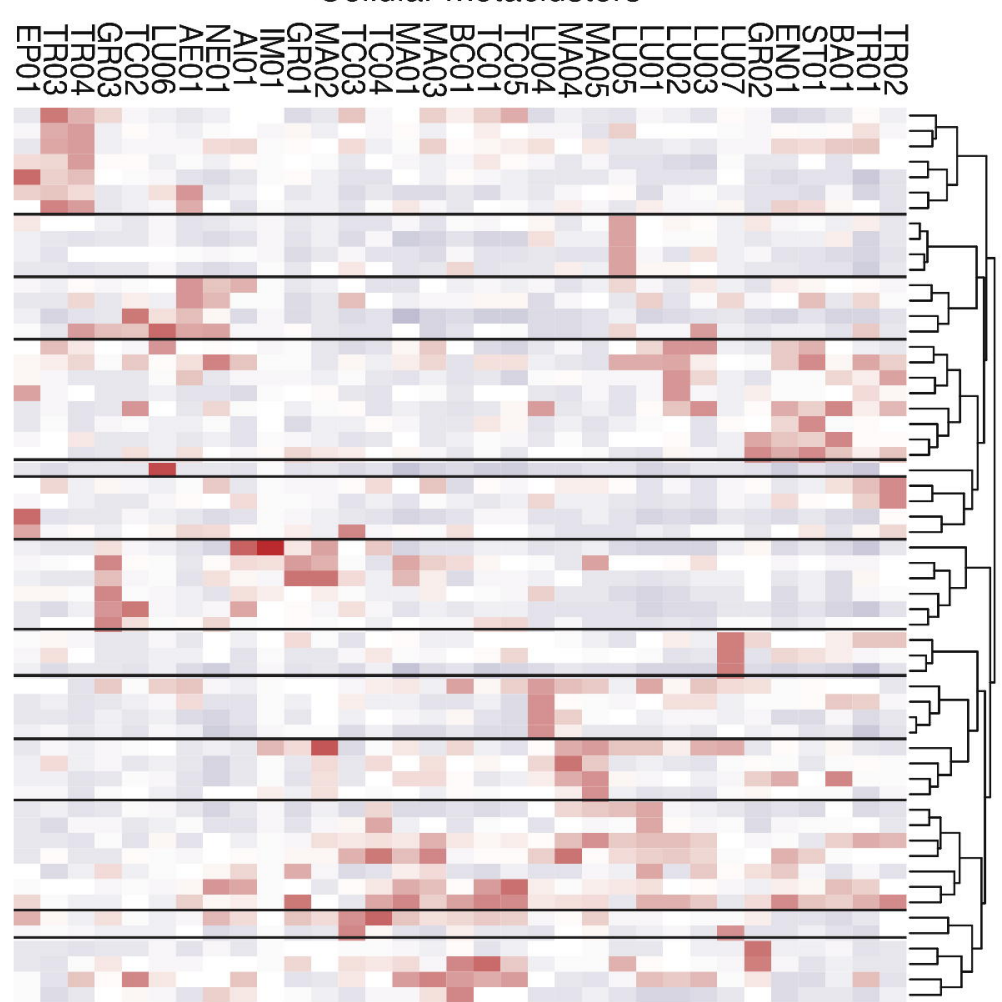

